University of Wollongong

Research Online

Faculty of Engineering and Information

Faculty of Engineering and Information

Sciences - Papers: Part A

Sciences

$1-1-2016$

Three-dimensional nonlinear global dynamics of axially moving viscoelastic beams

Hamed Farokhi

McGill University

Mergen $\mathrm{H}$. Ghayesh

University of Wollongong, mergen@uow.edu.au

Shahid Hussain

University of Wollongong, shussain@uow.edu.au

Follow this and additional works at: https://ro.uow.edu.au/eispapers

Part of the Engineering Commons, and the Science and Technology Studies Commons

Research Online is the open access institutional repository for the University of Wollongong. For further information contact the UOW Library: research-pubs@uow.edu.au 


\title{
Three-dimensional nonlinear global dynamics of axially moving viscoelastic beams
}

\begin{abstract}
The three-dimensional nonlinear global dynamics of an axially moving viscoelastic beam is investigated numerically, retaining longitudinal, transverse, and lateral displacements and inertia. The nonlinear continuous model governing the motion of the system is obtained by means of Hamilton's principle. The Galerkin scheme along with suitable eigenfunctions is employed for model reduction. Direct timeintegration is conducted upon the reduced-order model yielding the time-varying generalized coordinates. From the time histories of the generalized coordinates, the bifurcation diagrams of Poincaré sections are constructed by varying either the forcing amplitude or the axial speed as the bifurcation parameter. The results for the three-dimensional viscoelastic model are compared to those of a three-dimensional elastic model in order to better understand the effect of the internal energy dissipation mechanism on the dynamical behavior of the system. The results are also presented by means of time histories, phase-plane diagrams, and fast Fourier transforms (FFT).
\end{abstract}

\section{Keywords}

dimensional, nonlinear, global, dynamics, three, axially, moving, viscoelastic, beams

\section{Disciplines}

Engineering | Science and Technology Studies

\section{Publication Details}

Farokhi, H., Ghayesh, M. H. \& Hussain, S. (2016). Three-dimensional nonlinear global dynamics of axially moving viscoelastic beams. Journal of Vibration and Acoustics, Transactions of the ASME, 138 (1), 011007-1-011007-11. 


\section{Three-Dimensional Nonlinear Global Dynamics of Axially Moving Viscoelastic Beams}

Montreal, QC H3A 0C3, Canada e-mail: hamed.farokhi@mail.mcgill.ca

\author{
Mergen H. Ghayesh ${ }^{1}$ \\ School of Mechanical, \\ Materials and Mechatronic Engineering, \\ University of Wollongong, \\ Wollongong, New South Wales 2522, Australia \\ e-mail: mergen@uow.edu.au \\ Shahid Hussain \\ School of Mechanical, \\ Materials and Mechatronic Engineering. \\ University of Wollongong, \\ Wollongong, New South Wales 2522, Australia \\ e-mail: shussain@uow.edu.au
}

The three-dimensional nonlinear global dynamics of an axially moving viscoelastic beam is investigated numerically, retaining longitudinal, transverse, and lateral displacements and inertia. The nonlinear continuous model governing the motion of the system is obtained by means of Hamilton's principle. The Galerkin scheme along with suitable eigenfunctions is employed for model reduction. Direct time-integration is conducted upon the reduced-order model yielding the time-varying generalized coordinates. From the time histories of the generalized coordinates, the bifurcation diagrams of Poincaré sections are constructed by varying either the forcing amplitude or the axial speed as the bifurcation parameter. The results for the three-dimensional viscoelastic model are compared to those of a three-dimensional elastic model in order to better understand the effect of the internal energy dissipation mechanism on the dynamical behavior of the system. The results are also presented by means of time histories, phase-plane diagrams, and fast Fourier transforms (FFT). [DOI: 10.1115/1.4031600]

Keywords: three-dimensional axially moving beams, viscoelastic, nonlinear dynamics, bifurcation, global dynamics

\section{Introduction}

Axially moving systems are available in a large class of mechanical and industrial systems. The examples of which are cable tramways, robotic arms, textile fibers, band saw blades, magnetic tapes, and conveyor belts, to name a few. Investigations regarding the nonlinear dynamics of axially moving systems have been carried out for several decades, which are mainly due to their widespread applications as well as their interesting and complex dynamical behavior, especially when the axial speed is high.

In the modeling of axially moving systems, an important issue is how to model the material of the system [1-3]; Is an elastic or a viscoelastic [4,5] constitutive relation used? In fact, in many applications, employing an elastic constitutive relation does not yield accurate results. Therefore, taking into account an internal energy dissipation mechanism is important from both the qualitative and quantitative perspectives. Moreover, for the case of the Kelvin-Voigt energy dissipation mechanism, the steady dissipation effect, which is generated by employing a material derivative in the constitutive relation, affects the dynamic behavior of the system considerably, especially at high axial speeds.

In order to review the literature (on the dynamics of axially moving beams and strings) more effectively, two categories are defined. The first category consists of the investigations in which the planar dynamics of the system is examined, neglecting the lateral displacement [6,7]; in particular, either the transverse or the coupled longitudinal-transverse [8] dynamics of the system is examined. In the second category of the investigations, on the other hand, the lateral displacement as well as the longitudinal and transverse displacements are taken into account, i.e., a fully three-dimensional model is employed.

Starting with the first category, which includes most of the investigations in the literature, Mote [9] examined the dynamics of a band saw by obtaining the exact solution for the transverse dynamics and natural frequencies. Wickert [10] examined the free nonlinear dynamics of an axially moving tensioned beam in the

\footnotetext{
${ }^{1}$ Corresponding author.

Contributed by the Technical Committee on Vibration and Sound of ASME for publication in the Journal OF Vibration and Acoustics. Manuscript received February 25, 2015; final manuscript received September 11, 2015; published online October 26, 2015. Assoc. Editor: Izhak Bucher.
}

sub- and super-critical axial speed regimes. More advanced analytical techniques, such as the method of multiple scales and matched asymptotic expansion, were used by Pakdemirli and coworkers [11-13] in order to analyze the nonlinear dynamics of axially accelerating beams and strings. Marynowski and Kapitaniak [14] investigated the nonlinear dynamics of an axially moving viscoelastic beam by introducing a three-parameter Zener internal damping in the beam model; they used the Galerkin scheme along with Runge-Kutta method to solve the equations numerically. A finite element approach was used by Stylianou and Tabarrok [15], who analyzed the stability of an axially moving beam. Chakraborty and Mallik [16] examined the stability of an axially moving beam analytically by means of the method of multiple scales. Chen and coworkers [17-22] contributed to the field by examining the natural frequencies, steady-state responses, stability, and nonlinear dynamics of axially moving beams and strings with either constant or time-dependent axial speeds via employing both elastic and viscoelastic constitutive relations. Ravindra and Zhu [23] utilized a single-mode Galerkin approximation so as to examine the nonlinear dynamics of an axially moving continuum both analytically and numerically.

The literature regarding the second class of analysis, compared to the first class, is rather limited. Huang et al. [24] studied the dynamic stability of an axially moving string with time-varying tension, undergoing three-dimensional motions; they used the Galerkin scheme to discretize the equations and solved the equations employing the method of multiple scales. Chen et al. [25] contributed to the field by examining the nonlinear dynamics of an axially accelerated viscoelastic beam numerically taking into account the transverse and lateral displacements; the longitudinal displacement was neglected and three modes were considered for the transverse and lateral motions, resulting in a six degrees-offreedom system.

The present study, for the first time, investigates the nonlinear global dynamics of an axially moving viscoelastic beam taking into account the longitudinal, transverse, and lateral displacements and inertia (i.e., a three-dimensional model is considered). The Kelvin-Voigt viscoelastic model is employed in the constitutive relation. A high-dimensional system is considered by assuming a large number of modes in the Galerkin expansion, which enables to investigate the effect of higher harmonics on the nonlinear 
dynamic behavior of the system. In order to be able to solve this large set of equations, the computer codes should be welloptimized for both run-time and accuracy. Moreover, the bifurcation diagrams obtained by employing the viscoelastic model are compared to those obtained for the elastic system so as to highlight the effect of the internal energy dissipation mechanism on the global dynamics of the system.

\section{Continuous and Reduced-Order Models}

An axially moving viscoelastic beam with hinged-hinged supports is shown in Fig. 1. $L, \rho, E$, and $A$ represent the length, mass density, Young's modulus, and the area of the cross section of the beam, respectively. It is assumed that the beam is moving at a constant speed $c$ in the $x$ direction and subjected to distributed harmonic excitation loads in the transverse and lateral directions, i.e., $F_{1}(x) \cos (\omega t)$ and $F_{2}(x) \cos (\omega t)$, respectively, where $\omega$ is the excitation frequency and $y$ and $z$ directions are called the transverse and lateral directions, respectively. The axial coordinate is shown by $x$; $u(x, t), w(x, t)$, and $v(x, t)$ represent the longitudinal, transverse, and lateral displacements, in the $x, y$, and $z$ directions, respectively.

The nonlinear partial differential equations of motion are derived under the following assumptions: (1) the Euler-Bernoulli beam theory [26-28] is employed neglecting the effect of shear deformation and rotary inertia; (2) the stretching effect of the midplane of the beam (which is caused due to large deflections) is the source of geometric nonlinearity [29-34]; (3) the material, not partial, time derivative is used in the viscoelastic constitutive relation; (4) the beam is under a pretension $p$ in the axial direction; (5) the cross section of the beam is uniform along the entire length; (6) the rotation of beam cross section is assumed to be small [35]; and (7) the axial speed is constant [36].

The nonlinear strain-displacement relation for a threedimensional Euler-Bernoulli beam can be expressed as

$$
\varepsilon_{x}=\frac{\partial u}{\partial x}+\frac{1}{2}\left(\frac{\partial v}{\partial x}\right)^{2}+\frac{1}{2}\left(\frac{\partial w}{\partial x}\right)^{2}-z \frac{\partial^{2} v}{\partial x^{2}}-y \frac{\partial^{2} w}{\partial x^{2}}
$$

where $\varepsilon_{x}$ denotes the axial strain of a generic point of the beam located at distances $y$ and $z$ (in the $y$ and $z$ directions) from the centerline of the beam.

The constitutive relation for the Kelvin-Voigt energy dissipation mechanism, which is a two-parameter viscoelastic rheological model of the material [37], can be written as

$$
\sigma_{x}=E \varepsilon_{x}+\eta\left(\frac{\partial \varepsilon_{x}}{\partial t}+c \frac{\partial \varepsilon_{x}}{\partial x}\right)=\sigma_{x(e)}+\sigma_{x(v)}
$$

in which $\sigma_{x(e)}$ represents the elastic part of the stress while $\sigma_{x(v)}$ denotes the viscoelastic counterpart and $\eta$ is the material viscosity coefficient.

The potential energy of the system, which consists of a part due to the pretension in the axial direction and another part due to the elastic stress $\left(\sigma_{x(e)}\right)$, can be written as

$$
\begin{aligned}
U= & \int_{0}^{L}\left\{p\left[\frac{\partial u}{\partial x}+\frac{1}{2}\left(\frac{\partial v}{\partial x}\right)^{2}+\frac{1}{2}\left(\frac{\partial w}{\partial x}\right)^{2}\right]\right. \\
& \left.+\frac{1}{2} E A\left[\frac{\partial u}{\partial x}+\frac{1}{2}\left(\frac{\partial v}{\partial x}\right)^{2}+\frac{1}{2}\left(\frac{\partial w}{\partial x}\right)^{2}\right]\right\} \mathrm{d} x \\
& +\frac{1}{2} \int_{0}^{L} E\left[I_{y}\left(\frac{\partial^{2} v}{\partial x^{2}}\right)^{2}+I_{z}\left(\frac{\partial^{2} w}{\partial x^{2}}\right)^{2}\right] \mathrm{d} x
\end{aligned}
$$

in which $I_{y}$ and $I_{z}$ represent the second moments of area about the $y$ and $z$ axes, respectively, and $p$ represents the axial tension in the beam.

The kinetic energy of the system can be formulated as

$$
\begin{aligned}
T= & \frac{1}{2} \rho A \int_{0}^{L}\left\{\left[\frac{\partial u}{\partial t}+c\left(1+\frac{\partial u}{\partial x}\right)\right]^{2}+\left(\frac{\partial v}{\partial t}+c \frac{\partial v}{\partial x}\right)^{2}\right. \\
& \left.+\left(\frac{\partial w}{\partial t}+c \frac{\partial w}{\partial x}\right)^{2}\right\} \mathrm{d} x
\end{aligned}
$$

The variation of the work done by the viscoelastic stress $\left(\sigma_{x(v)}\right)$ can be expressed as

$$
\delta W_{\text {vis }}=\int_{V} \sigma_{x(v)} \delta \varepsilon_{x} \mathrm{~d} V=\int_{0}^{L} \int_{A} \sigma_{x(v)} \delta \varepsilon_{x} \mathrm{~d} A \mathrm{~d} x
$$

The variation of the work done by the external harmonic excitation loads and the viscous damping due to surrounding medium moving at the same speed of the beam (with the damping coefficient of $\mu$ ) can be written as

$$
\begin{gathered}
\delta W_{F}=\int_{0}^{L} F_{1}(x) \cos (\omega t) \delta w \mathrm{~d} x+\int_{0}^{L} F_{2}(x) \cos (\omega t) \delta v \mathrm{~d} x \\
\delta W_{D}=-\mu \int_{0}^{L}\left(\frac{\partial u}{\partial t} \delta u+\frac{\partial v}{\partial t} \delta v+\frac{\partial w}{\partial t} \delta w\right) \mathrm{d} x
\end{gathered}
$$

Substitution of Eqs. (3)-(7) into the extended Hamilton's principle results in the following dimensionless nonlinear partial differential equations governing the motion of the system in the longitudinal, transverse, and lateral directions, respectively,

$$
\begin{aligned}
\frac{\partial^{2} u}{\partial t^{2}} & +2 c \frac{\partial^{2} u}{\partial x \partial t}+c^{2} \frac{\partial^{2} u}{\partial x^{2}}+\mu \frac{\partial u}{\partial t}=v_{1}^{2}\left(\frac{\partial^{2} u}{\partial x^{2}}+\frac{\partial v}{\partial x} \frac{\partial^{2} v}{\partial x^{2}}+\frac{\partial w}{\partial x} \frac{\partial^{2} w}{\partial x^{2}}\right) \\
& +\zeta\left(\frac{\partial^{3} u}{\partial x^{2} \partial t}+\frac{\partial^{2} v}{\partial x \partial t} \frac{\partial^{2} v}{\partial x^{2}}+\frac{\partial v}{\partial x} \frac{\partial^{3} v}{\partial x^{2} \partial t}+\frac{\partial^{2} w}{\partial x \partial t} \frac{\partial^{2} w}{\partial x^{2}}+\frac{\partial w}{\partial x} \frac{\partial^{3} w}{\partial x^{2} \partial t}\right) \\
& +\zeta c\left[\frac{\partial^{3} u}{\partial x^{3}}+\left(\frac{\partial^{2} v}{\partial x^{2}}\right)^{2}+\frac{\partial v}{\partial x} \frac{\partial^{3} v}{\partial x^{3}}+\left(\frac{\partial^{2} w}{\partial x^{2}}\right)^{2}+\frac{\partial w}{\partial x} \frac{\partial^{3} w}{\partial x^{3}}\right]
\end{aligned}
$$

$$
\begin{aligned}
& \frac{\partial^{2} w}{\partial t^{2}}+2 c \frac{\partial^{2} w}{\partial x \partial t}+\left(c^{2}-1\right) \frac{\partial^{2} w}{\partial x^{2}}+v_{f}^{2} \frac{\partial^{4} w}{\partial x^{4}}+\gamma\left(\frac{\partial^{5} w}{\partial x^{4} \partial t}+c \frac{\partial^{5} w}{\partial x^{5}}\right)+\mu \frac{\partial w}{\partial t} \\
& =v_{1}^{2}\left[\frac{\partial u}{\partial x} \frac{\partial^{2} w}{\partial x^{2}}+\frac{\partial^{2} u}{\partial x^{2}} \frac{\partial w}{\partial x}+\frac{\partial w}{\partial x} \frac{\partial v}{\partial x} \frac{\partial^{2} v}{\partial x^{2}}+\frac{1}{2}\left(\frac{\partial v}{\partial x}\right)^{2} \frac{\partial^{2} w}{\partial x^{2}}+\frac{3}{2}\left(\frac{\partial w}{\partial x}\right)^{2} \frac{\partial^{2} w}{\partial x^{2}}\right] \\
& +\zeta\left[\frac{\partial^{2} u}{\partial x \partial t} \frac{\partial^{2} w}{\partial x^{2}}+\frac{\partial^{3} u}{\partial x^{2} \partial t} \frac{\partial w}{\partial x}+\frac{\partial w}{\partial x} \frac{\partial^{2} v}{\partial x \partial t} \frac{\partial^{2} v}{\partial x^{2}}+\frac{\partial w}{\partial x} \frac{\partial v}{\partial x} \frac{\partial^{3} v}{\partial x^{2} \partial t}+\frac{\partial v}{\partial x} \frac{\partial^{2} v}{\partial x \partial t} \frac{\partial^{2} w}{\partial x^{2}}+\left(\frac{\partial w}{\partial x}\right)^{2} \frac{\partial^{3} w}{\partial x^{2} \partial t}+2 \frac{\partial w}{\partial x} \frac{\partial^{2} w}{\partial x \partial t} \frac{\partial^{2} w}{\partial x^{2}}\right] \\
& +\zeta c\left[\frac{\partial^{2} u}{\partial x^{2}} \frac{\partial^{2} w}{\partial x^{2}}+\frac{\partial^{3} u}{\partial x^{3}} \frac{\partial w}{\partial x}+\frac{\partial w}{\partial x}\left(\frac{\partial^{2} v}{\partial x^{2}}\right)^{2}+\frac{\partial w}{\partial x} \frac{\partial v}{\partial x} \frac{\partial^{3} v}{\partial x^{3}}+\frac{\partial v}{\partial x} \frac{\partial^{2} v}{\partial x^{2}} \frac{\partial^{2} w}{\partial x^{2}}+\left(\frac{\partial w}{\partial x}\right)^{2} \frac{\partial^{3} w}{\partial x^{3}}+2 \frac{\partial w}{\partial x}\left(\frac{\partial^{2} w}{\partial x^{2}}\right)^{2}\right]+F_{1}(x) \cos (\omega t)
\end{aligned}
$$




$$
\begin{aligned}
& \frac{\partial^{2} v}{\partial t^{2}}+2 c \frac{\partial^{2} v}{\partial x \partial t}+\left(c^{2}-1\right) \frac{\partial^{2} v}{\partial x^{2}}+\alpha^{2} v_{f}^{2} \frac{\partial^{4} v}{\partial x^{4}}+\alpha^{2} \gamma\left(\frac{\partial^{5} v}{\partial x^{4} \partial t}+c \frac{\partial^{5} v}{\partial x^{5}}\right)+\mu \frac{\partial v}{\partial t} \\
& =v_{1}^{2}\left[\frac{\partial u}{\partial x} \frac{\partial^{2} v}{\partial x^{2}}+\frac{\partial^{2} u}{\partial x^{2}} \frac{\partial v}{\partial x}+\frac{\partial v}{\partial x} \frac{\partial w}{\partial x} \frac{\partial^{2} w}{\partial x^{2}}+\frac{1}{2}\left(\frac{\partial w}{\partial x}\right)^{2} \frac{\partial^{2} v}{\partial x^{2}}+\frac{3}{2}\left(\frac{\partial v}{\partial x}\right)^{2} \frac{\partial^{2} v}{\partial x^{2}}\right] \\
& +\zeta\left[\frac{\partial^{2} u}{\partial x \partial t} \frac{\partial^{2} v}{\partial x^{2}}+\frac{\partial^{3} u}{\partial x^{2} \partial t} \frac{\partial v}{\partial x}+\frac{\partial v}{\partial x} \frac{\partial^{2} w}{\partial x \partial t} \frac{\partial^{2} w}{\partial x^{2}}+\frac{\partial v}{\partial x} \frac{\partial w}{\partial x} \frac{\partial^{3} w}{\partial x^{2} \partial t}+\frac{\partial w}{\partial x} \frac{\partial^{2} w}{\partial x \partial t} \frac{\partial^{2} v}{\partial x^{2}}+\left(\frac{\partial v}{\partial x}\right)^{2} \frac{\partial^{3} v}{\partial x^{2} \partial t}+2 \frac{\partial v}{\partial x} \frac{\partial^{2} v}{\partial x \partial t} \frac{\partial^{2} v}{\partial x^{2}}\right] \\
& +\zeta c\left[\frac{\partial^{2} u}{\partial x^{2}} \frac{\partial^{2} v}{\partial x^{2}}+\frac{\partial^{3} u}{\partial x^{3}} \frac{\partial v}{\partial x}+\frac{\partial v}{\partial x}\left(\frac{\partial^{2} w}{\partial x^{2}}\right)^{2}+\frac{\partial v}{\partial x} \frac{\partial w}{\partial x} \frac{\partial^{3} w}{\partial x^{3}}+\frac{\partial w}{\partial x} \frac{\partial^{2} w}{\partial x^{2}} \frac{\partial^{2} v}{\partial x^{2}}+\left(\frac{\partial v}{\partial x}\right)^{2} \frac{\partial^{3} v}{\partial x^{3}}+2 \frac{\partial v}{\partial x}\left(\frac{\partial^{2} v}{\partial x^{2}}\right)^{2}\right]+F_{2}(x) \cos (\omega t)
\end{aligned}
$$

where following dimensionless parameters are used and the asterisk notation is dropped for briefness:

$$
\begin{aligned}
u^{*} & =\frac{u}{L}, \quad w^{*}=\frac{w}{L}, \quad v^{*}=\frac{v}{L}, \quad x^{*}=\frac{x}{L}, \quad t^{*}=t \sqrt{\frac{p}{\rho A L^{2}}}, \quad c^{*}=c \sqrt{\frac{\rho A}{p}} \\
F_{1}^{*} & =\frac{F_{1} L}{p}, \quad F_{2}^{*}=\frac{F_{2} L}{p}, \quad v_{f}=\sqrt{\frac{E I_{z}}{p L^{2}}}, \quad \Omega=\omega \sqrt{\frac{\rho A L^{2}}{p}}, \quad v_{1}=\sqrt{\frac{E A}{p}} \\
\alpha & =\sqrt{\frac{I_{y}}{I_{z}}}, \quad \mu^{*}=\frac{\mu L}{p} \sqrt{\frac{p}{\rho A}}, \quad \gamma=\frac{\eta I_{z}}{p L^{3}} \sqrt{\frac{p}{\rho A}}, \quad \zeta=\frac{\eta A}{p L} \sqrt{\frac{p}{\rho A}}
\end{aligned}
$$

Those terms multiplied by $\zeta c$ in Eqs. (8)-(10) are called steady dissipation terms, which are due to the simultaneous presence of the axial speed and internal energy dissipation mechanism in the system and do not appear in stationary systems-these terms become more dominant at high axial speeds. The dimensionless boundary conditions can be expressed as

$$
\begin{aligned}
& \left.u\right|_{x=0}=\left.u\right|_{x=1}=0,\left.\quad w\right|_{x=0}=\left.w\right|_{x=1}=0,\left.\quad \frac{\partial^{2} w}{\partial x^{2}}\right|_{x=0}=\left.\frac{\partial^{2} w}{\partial x^{2}}\right|_{x=1}=0 \\
& \left.v\right|_{x=0}=\left.v\right|_{x=1}=0,\left.\quad \frac{\partial^{2} v}{\partial x^{2}}\right|_{x=0}=\left.\frac{\partial^{2} v}{\partial x^{2}}\right|_{x=1}=0
\end{aligned}
$$

The motions of the system are defined by the following approximate series expansions:

$$
w(x, t)=\sum_{i=1}^{M} \phi_{i}(x) q_{i}(t), \quad v(x, t)=\sum_{i=1}^{N} \phi_{i}(x) p_{i}(t), \quad u(x, t)=\sum_{i=1}^{Q} \phi_{i}(x) r_{i}(t)
$$

in which $\phi_{i}(x)$ represents the $i$ th eigenfunction for the transverse motion of a hinged-hinged linear stationary beam and $q_{i}(t), p_{i}(t)$, and $r_{i}(t)$ denote the $i$ th generalized coordinate for the transverse, lateral, and longitudinal motions, respectively.

The Galerkin method is applied to the equations of motion by substituting Eq. (13) into Eqs. (8)-(10), choosing $F_{1}(x)=f_{1} \phi_{1}(x)$ and $F_{2}(x)=f_{2} \phi_{1}(x)$, multiplying the resultant equations by the corresponding eigenfunctions, and then integrating with respect to $x$ from zero to one. This procedure yields a set of $(M+N+Q)$ second-order nonlinear ordinary differential equations with coupled nonlinear terms as

$$
\begin{aligned}
& \sum_{j=1}^{Q}\left(\int_{0}^{1} \phi_{i} \phi_{j} \mathrm{~d} x\right) \ddot{r}_{j}+2 c \sum_{j=1}^{Q}\left(\int_{0}^{1} \phi_{i} \phi_{j}^{\prime} \mathrm{d} x\right) \dot{r}_{j}+c^{2} \sum_{j=1}^{Q}\left(\int_{0}^{1} \phi_{i} \phi_{j}^{\prime \prime} \mathrm{d} x\right) r_{j}+\mu \sum_{j=1}^{Q}\left(\int_{0}^{1} \phi_{i} \phi_{j} \mathrm{~d} x\right) \dot{r}_{j} \\
& \quad=v_{1}^{2}\left[\sum_{j=1}^{Q}\left(\int_{0}^{1} \phi_{i} \phi_{j}^{\prime \prime} \mathrm{d} x\right) r_{j}+\sum_{j=1}^{N} \sum_{k=1}^{N}\left(\int_{0}^{1} \phi_{i} \phi_{j}^{\prime} \phi_{k}^{\prime \prime} \mathrm{d} x\right) p_{j} p_{k}+\sum_{j=1}^{M} \sum_{k=1}^{M}\left(\int_{0}^{1} \phi_{i} \phi_{j}^{\prime} \phi_{k}^{\prime \prime} \mathrm{d} x\right) q_{j} q_{k}\right] \\
& \quad+\zeta\left[\sum_{j=1}^{Q}\left(\int_{0}^{1} \phi_{i} \phi_{j}^{\prime \prime} \mathrm{d} x\right) \dot{r}_{j}+\sum_{j=1}^{N} \sum_{k=1}^{N}\left(\int_{0}^{1} \phi_{i} \phi_{j}^{\prime} \phi_{k}^{\prime \prime} \mathrm{d} x\right) \dot{p}_{j} p_{k}+\sum_{j=1}^{N} \sum_{k=1}^{N}\left(\int_{0}^{1} \phi_{i} \phi_{j}^{\prime} \phi_{k}^{\prime \prime} \mathrm{d} x\right) p_{j} \dot{p}_{k}+\sum_{j=1}^{M} \sum_{k=1}^{M}\left(\int_{0}^{1} \phi_{i} \phi_{j}^{\prime} \phi_{k}^{\prime \prime} \mathrm{d} x\right) \dot{q}_{j} q_{k}\right. \\
& \left.\quad+\sum_{j=1}^{M} \sum_{k=1}^{M}\left(\int_{0}^{1} \phi_{i} \phi_{j}^{\prime} \phi_{k}^{\prime \prime} \mathrm{d} x\right) q_{j} \dot{q}_{k}\right]+\zeta c\left[\sum_{j=1}^{Q}\left(\int_{0}^{1} \phi_{i} \phi_{j}^{\prime \prime \prime} \mathrm{d} x\right) r_{j}+\sum_{j=1}^{N} \sum_{k=1}^{N}\left(\int_{0}^{1} \phi_{i} \phi_{j}^{\prime \prime} \phi_{k}^{\prime \prime} \mathrm{d} x\right) p_{j} p_{k}\right. \\
& \left.\quad+\sum_{j=1}^{N} \sum_{k=1}^{N}\left(\int_{0}^{1} \phi_{i} \phi_{j}^{\prime} \phi_{k}^{\prime \prime \prime} \mathrm{d} x\right) p_{j} p_{k}+\sum_{j=1}^{M} \sum_{k=1}^{M}\left(\int_{0}^{1} \phi_{i} \phi_{j}^{\prime \prime} \phi_{k}^{\prime \prime} \mathrm{d} x\right) q_{j} q_{k}+\sum_{j=1}^{M} \sum_{k=1}^{M}\left(\int_{0}^{1} \phi_{i} \phi_{j}^{\prime} \phi_{k}^{\prime \prime \prime} \mathrm{d} x\right) q_{j} q_{k}\right], \quad i=1,2, \ldots, Q
\end{aligned}
$$




$$
\begin{aligned}
& \sum_{j=1}^{M}\left(\int_{0}^{1} \phi_{i} \phi_{j} \mathrm{~d} x\right) \ddot{q}_{j}+2 c \sum_{j=1}^{M}\left(\int_{0}^{1} \phi_{i} \phi_{j}^{\prime} \mathrm{d} x\right) \dot{q}_{j}+\left(c^{2}-1\right) \sum_{j=1}^{M}\left(\int_{0}^{1} \phi_{i} \phi_{j}^{\prime \prime} \mathrm{d} x\right) q_{j}+\mu \sum_{j=1}^{M}\left(\int_{0}^{1} \phi_{i} \phi_{j} \mathrm{~d} x\right) \dot{q}_{j}+v_{f}^{2} \sum_{j=1}^{M}\left(\int_{0}^{1} \phi_{i} \phi_{j}^{\prime \prime \prime \prime} \mathrm{d} x\right) q_{j} \\
& +\gamma c \sum_{j=1}^{M}\left(\int_{0}^{1} \phi_{i} \phi_{j}^{\prime \prime \prime \prime \prime} \mathrm{d} x\right) q_{j}+\gamma \sum_{j=1}^{M}\left(\int_{0}^{1} \phi_{i} \phi_{j}^{\prime \prime \prime \prime} \mathrm{d} x\right) \dot{q}_{j} \\
& =v_{1}^{2}\left[\sum_{j=1}^{Q} \sum_{k=1}^{M}\left(\int_{0}^{1} \phi_{i} \phi_{j}^{\prime} \phi_{k}^{\prime \prime} \mathrm{d} x\right) r_{j} q_{k}+\sum_{j=1}^{Q} \sum_{k=1}^{M}\left(\int_{0}^{1} \phi_{i} \phi_{j}^{\prime \prime} \phi_{k}^{\prime} \mathrm{d} x\right) r_{j} q_{k}+\sum_{j=1}^{M} \sum_{k=1}^{N} \sum_{l=1}^{N}\left(\int_{0}^{1} \phi_{i} \phi_{j}^{\prime} \phi_{k}^{\prime} \phi_{l}^{\prime \prime} \mathrm{d} x\right) q_{j} p_{k} p_{l}\right. \\
& \left.+\frac{1}{2} \sum_{j=1}^{N} \sum_{k=1}^{N} \sum_{l=1}^{M}\left(\int_{0}^{1} \phi_{i} \phi_{j}^{\prime} \phi_{k}^{\prime} \phi_{l}^{\prime \prime} \mathrm{d} x\right) p_{j} p_{k} q_{l}+\frac{3}{2} \sum_{j=1}^{M} \sum_{k=1}^{M} \sum_{l=1}^{M}\left(\int_{0}^{1} \phi_{i} \phi_{j}^{\prime} \phi_{k}^{\prime} \phi_{l}^{\prime \prime} \mathrm{d} x\right) q_{j} q_{k} q_{l}\right]+\zeta\left[\sum_{j=1}^{Q} \sum_{k=1}^{M}\left(\int_{0}^{1} \phi_{i} \phi_{j}^{\prime} \phi_{k}^{\prime \prime} \mathrm{d} x\right) \dot{r}_{j} q_{k}\right. \\
& +\sum_{j=1}^{Q} \sum_{k=1}^{M}\left(\int_{0}^{1} \phi_{i} \phi_{j}^{\prime \prime} \phi_{k}^{\prime} \mathrm{d} x\right) \dot{r}_{j} q_{k}+\sum_{j=1}^{M} \sum_{k=1}^{N} \sum_{l=1}^{N}\left(\int_{0}^{1} \phi_{i} \phi_{j}^{\prime} \phi_{k}^{\prime} \phi_{l}^{\prime \prime} \mathrm{d} x\right) q_{j} \dot{p}_{k} p_{l}+\sum_{j=1}^{M} \sum_{k=1}^{N} \sum_{l=1}^{N}\left(\int_{0}^{1} \phi_{i} \phi_{j}^{\prime} \phi_{k}^{\prime} \phi_{l}^{\prime \prime} \mathrm{d} x\right) q_{j} p_{k} \dot{p}_{l} \\
& \left.+\sum_{j=1}^{N} \sum_{k=1}^{N} \sum_{l=1}^{M}\left(\int_{0}^{1} \phi_{i} \phi_{j}^{\prime} \phi_{k}^{\prime} \phi_{l}^{\prime \prime} \mathrm{d} x\right) p_{j} \dot{p}_{k} q_{l}+\sum_{j=1}^{M} \sum_{k=1}^{M} \sum_{l=1}^{M}\left(\int_{0}^{1} \phi_{i} \phi_{j}^{\prime} \phi_{k}^{\prime} \phi_{l}^{\prime \prime} \mathrm{d} x\right) q_{j} q_{k} \dot{q}_{l}+2 \sum_{j=1}^{M} \sum_{k=1}^{M} \sum_{l=1}^{M}\left(\int_{0}^{1} \phi_{i} \phi_{j}^{\prime} \phi_{k}^{\prime} \phi_{l}^{\prime \prime} \mathrm{d} x\right) q_{j} \dot{q}_{k} q_{l}\right] \\
& +\zeta c\left[\sum_{j=1}^{Q} \sum_{k=1}^{M}\left(\int_{0}^{1} \phi_{i} \phi_{j}^{\prime \prime} \phi_{k}^{\prime \prime} \mathrm{d} x\right) r_{j} q_{k}+\sum_{j=1}^{Q} \sum_{k=1}^{M}\left(\int_{0}^{1} \phi_{i} \phi_{j}^{\prime \prime \prime} \phi_{k}^{\prime} \mathrm{d} x\right) r_{j} q_{k}+\sum_{j=1}^{M} \sum_{k=1}^{N} \sum_{l=1}^{N}\left(\int_{0}^{1} \phi_{i} \phi_{j}^{\prime} \phi_{k}^{\prime \prime} \phi_{l}^{\prime \prime} \mathrm{d} x\right) q_{j} p_{k} p_{l}\right. \\
& +\sum_{j=1}^{M} \sum_{k=1}^{N} \sum_{l=1}^{N}\left(\int_{0}^{1} \phi_{i} \phi_{j}^{\prime} \phi_{k}^{\prime} \phi_{l}^{\prime \prime \prime} \mathrm{d} x\right) q_{j} p_{k} p_{l}+\sum_{j=1}^{N} \sum_{k=1}^{N} \sum_{l=1}^{M}\left(\int_{0}^{1} \phi_{i} \phi_{j}^{\prime} \phi_{k}^{\prime \prime} \phi_{l}^{\prime \prime} \mathrm{d} x\right) p_{j} p_{k} q_{l}+\sum_{j=1}^{M} \sum_{k=1}^{M} \sum_{l=1}^{M}\left(\int_{0}^{1} \phi_{i} \phi_{j}^{\prime} \phi_{k}^{\prime} \phi_{l}^{\prime \prime \prime} \mathrm{d} x\right) q_{j} q_{k} q_{l} \\
& \left.+2 \sum_{j=1}^{M} \sum_{k=1}^{M} \sum_{l=1}^{M}\left(\int_{0}^{1} \phi_{i} \phi_{j}^{\prime} \phi_{k}^{\prime \prime} \phi_{l}^{\prime \prime} \mathrm{d} x\right) q_{j} q_{k} q_{l}\right]+\int_{0}^{1} f_{1} \phi_{1} \phi_{i} \mathrm{~d} x \cos (\Omega t), \quad i=1,2, \ldots, M
\end{aligned}
$$

$$
\begin{aligned}
& \sum_{j=1}^{N}\left(\int_{0}^{1} \phi_{i} \phi_{j} \mathrm{~d} x\right) \ddot{p}_{j}+2 c \sum_{j=1}^{N}\left(\int_{0}^{1} \phi_{i} \phi_{j}^{\prime} \mathrm{d} x\right) \dot{p}_{j}+\left(c^{2}-1\right) \sum_{j=1}^{N}\left(\int_{0}^{1} \phi_{i} \phi_{j}^{\prime \prime} \mathrm{d} x\right) p_{j} \\
& +\mu \sum_{j=1}^{N}\left(\int_{0}^{1} \phi_{i} \phi_{j} \mathrm{~d} x\right) \dot{p}_{j}+\alpha^{2} v_{f}^{2} \sum_{j=1}^{N}\left(\int_{0}^{1} \phi_{i} \phi_{j}^{\prime \prime \prime \prime} \mathrm{d} x\right) p_{j}+\alpha^{2} \gamma c \sum_{j=1}^{M}\left(\int_{0}^{1} \phi_{i} \phi_{j}^{\prime \prime \prime \prime \prime} \mathrm{d} x\right) p_{j} \\
& +\alpha^{2} \gamma \sum_{j=1}^{M}\left(\int_{0}^{1} \phi_{i} \phi_{j}^{\prime \prime \prime \prime} \mathrm{d} x\right) \dot{p}_{j}=v_{1}^{2}\left[\sum_{j=1}^{Q} \sum_{k=1}^{N}\left(\int_{0}^{1} \phi_{i} \phi_{j}^{\prime} \phi_{k}^{\prime \prime} \mathrm{d} x\right) r_{j} p_{k}+\sum_{j=1}^{Q} \sum_{k=1}^{N}\left(\int_{0}^{1} \phi_{i} \phi_{j}^{\prime \prime} \phi_{k}^{\prime} \mathrm{d} x\right) r_{j} p_{k}\right. \\
& +\sum_{j=1}^{N} \sum_{k=1}^{M} \sum_{l=1}^{M}\left(\int_{0}^{1} \phi_{i} \phi_{j}^{\prime} \phi_{k}^{\prime} \phi_{l}^{\prime \prime} \mathrm{d} x\right) p_{j} q_{k} q_{l}+\frac{1}{2} \sum_{j=1}^{M} \sum_{k=1}^{M} \sum_{l=1}^{N}\left(\int_{0}^{1} \phi_{i} \phi_{j}^{\prime} \phi_{k}^{\prime} \phi_{l}^{\prime \prime} \mathrm{d} x\right) q_{j} q_{k} p_{l} \\
& \left.+\frac{3}{2} \sum_{j=1}^{N} \sum_{k=1}^{N} \sum_{l=1}^{N}\left(\int_{0}^{1} \phi_{i} \phi_{j}^{\prime} \phi_{k}^{\prime} \phi_{l}^{\prime \prime} \mathrm{d} x\right) p_{j} p_{k} p_{l}\right]+\zeta\left[\sum_{j=1}^{Q} \sum_{k=1}^{N}\left(\int_{0}^{1} \phi_{i} \phi_{j}^{\prime} \phi_{k}^{\prime \prime} \mathrm{d} x\right) \dot{r}_{j} p_{k}+\sum_{j=1}^{Q} \sum_{k=1}^{N}\left(\int_{0}^{1} \phi_{i} \phi_{j}^{\prime \prime} \phi_{k}^{\prime} \mathrm{d} x\right) \dot{r}_{j} p_{k}\right. \\
& +\sum_{j=1}^{N} \sum_{k=1}^{M} \sum_{l=1}^{M}\left(\int_{0}^{1} \phi_{i} \phi_{j}^{\prime} \phi_{k}^{\prime} \phi_{l}^{\prime \prime} \mathrm{d} x\right) p_{j} \dot{q}_{k} q_{l}+\sum_{j=1}^{N} \sum_{k=1}^{M} \sum_{l=1}^{M}\left(\int_{0}^{1} \phi_{i} \phi_{j}^{\prime} \phi_{k}^{\prime} \phi_{l}^{\prime \prime} \mathrm{d} x\right) p_{j} q_{k} \dot{q}_{l}+\sum_{j=1}^{M} \sum_{k=1}^{M} \sum_{l=1}^{N}\left(\int_{0}^{1} \phi_{i} \phi_{j}^{\prime} \phi_{k}^{\prime} \phi_{l}^{\prime \prime} \mathrm{d} x\right) q_{j} \dot{q}_{k} p_{l} \\
& \left.+\sum_{j=1}^{N} \sum_{k=1}^{N} \sum_{l=1}^{N}\left(\int_{0}^{1} \phi_{i} \phi_{j}^{\prime} \phi_{k}^{\prime} \phi_{l}^{\prime \prime} \mathrm{d} x\right) p_{j} p_{k} \dot{p}_{l}+2 \sum_{j=1}^{N} \sum_{k=1}^{N} \sum_{l=1}^{N}\left(\int_{0}^{1} \phi_{i} \phi_{j}^{\prime} \phi_{k}^{\prime} \phi_{l}^{\prime \prime} \mathrm{d} x\right) p_{j} \dot{p}_{k} p_{l}\right]+\zeta c\left[\sum_{j=1}^{Q} \sum_{k=1}^{N}\left(\int_{0}^{1} \phi_{i} \phi_{j}^{\prime \prime} \phi_{k}^{\prime \prime} \mathrm{d} x\right) r_{j} p_{k}\right. \\
& +\sum_{j=1}^{Q} \sum_{k=1}^{N}\left(\int_{0}^{1} \phi_{i} \phi_{j}^{\prime \prime \prime} \phi_{k}^{\prime} \mathrm{d} x\right) r_{j} p_{k}+\sum_{j=1}^{N} \sum_{k=1}^{M} \sum_{l=1}^{M}\left(\int_{0}^{1} \phi_{i} \phi_{j}^{\prime} \phi_{k}^{\prime \prime} \phi_{l}^{\prime \prime} \mathrm{d} x\right) p_{j} q_{k} q_{l} \\
& +\sum_{j=1}^{N} \sum_{k=1}^{M} \sum_{l=1}^{M}\left(\int_{0}^{1} \phi_{i} \phi_{j}^{\prime} \phi_{k}^{\prime} \phi_{l}^{\prime \prime \prime} \mathrm{d} x\right) p_{j} q_{k} q_{l}+\sum_{j=1}^{M} \sum_{k=1}^{M} \sum_{l=1}^{N}\left(\int_{0}^{1} \phi_{i} \phi_{j}^{\prime} \phi_{k}^{\prime \prime} \phi_{l}^{\prime \prime} \mathrm{d} x\right) q_{j} q_{k} p_{l}+\sum_{j=1}^{N} \sum_{k=1}^{N} \sum_{l=1}^{N}\left(\int_{0}^{1} \phi_{i} \phi_{j}^{\prime} \phi_{k}^{\prime} \phi_{l}^{\prime \prime \prime} \mathrm{d} x\right) p_{j} p_{k} p_{l} \\
& \left.+2 \sum_{j=1}^{N} \sum_{k=1}^{N} \sum_{l=1}^{N}\left(\int_{0}^{1} \phi_{i} \phi_{j}^{\prime} \phi_{k}^{\prime \prime} \phi_{l}^{\prime \prime} \mathrm{d} x\right) p_{j} p_{k} p_{l}\right]+\int_{0}^{1} f_{2} \phi_{1} \phi_{i} \mathrm{~d} x \cos (\Omega t), \quad i=1,2, \ldots, N
\end{aligned}
$$

011007-4 / Vol. 138, FEBRUARY 2016

Transactions of the ASME

Downloaded From: http://vibrationacoustics.asmedigitalcollection.asme.org/ on 12/21/2015 Terms of Use: http://www.asme.org/about-asme/terms-of-use 


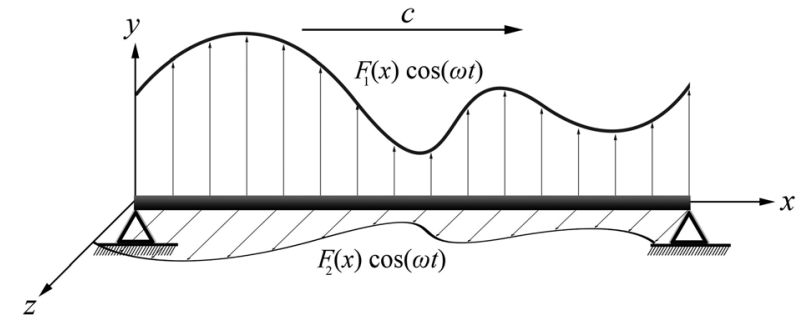

Fig. 1 Schematic representation of a three-dimensional axially moving viscoelastic beam

(a)

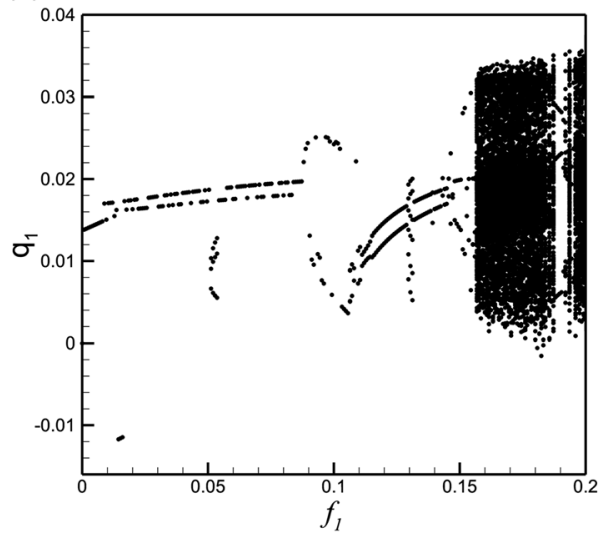

(c)

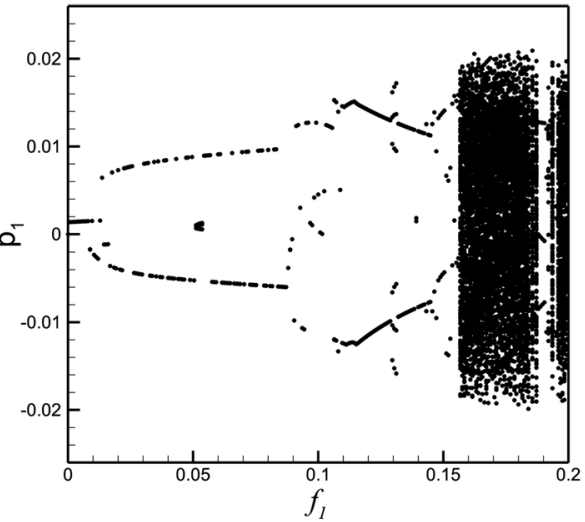

(e)

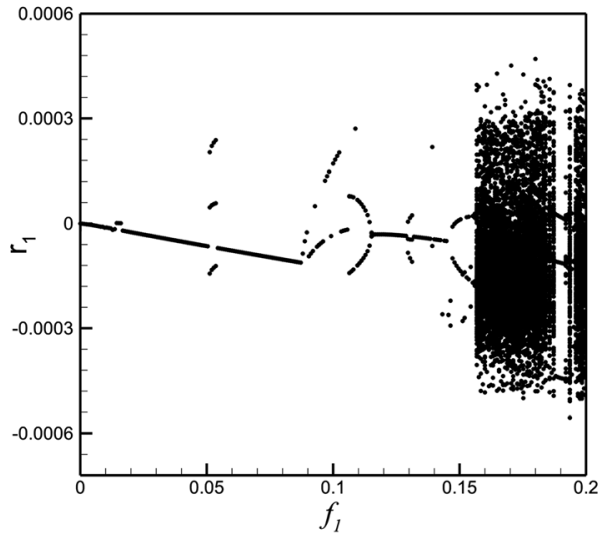

in which the dot and prime notations represent the differentiation with respect to the dimensionless time and axial coordinates, respectively.

Equations (14)-(16) are transformed into a new set of $2(M+N+Q)$ first-order nonlinear ordinary differential equations via the following substitutions: $x_{i}=\dot{q}_{i}, y_{i}=\dot{p}_{i}$, and $z_{i}=\dot{r}_{i}$. In the present study, $M=N=Q=6$ are chosen (i.e., an 18 degrees-offreedom system), resulting in 36 first-order nonlinear ordinary differential equations with coupled nonlinear terms; these values for $M, N$, and $Q$ are obtained by performing a convergence test analysis in order to find the sufficient number of modes required to obtain converged results. This set of equations is integrated numerically employing the modified Rosenbrock technique. This operation yields the time histories of the generalized coordinates

(b)

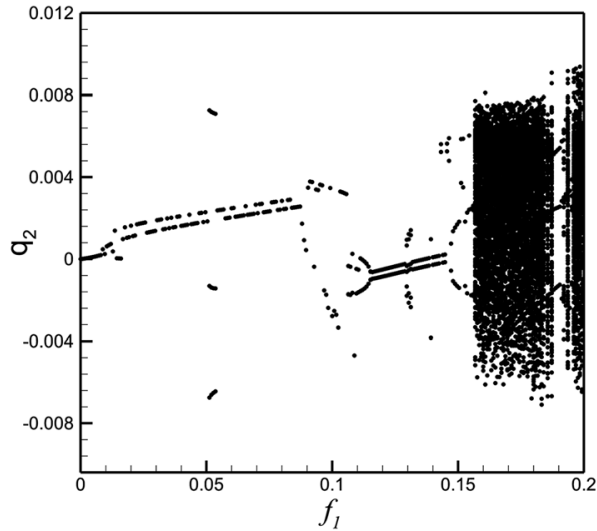

(d)

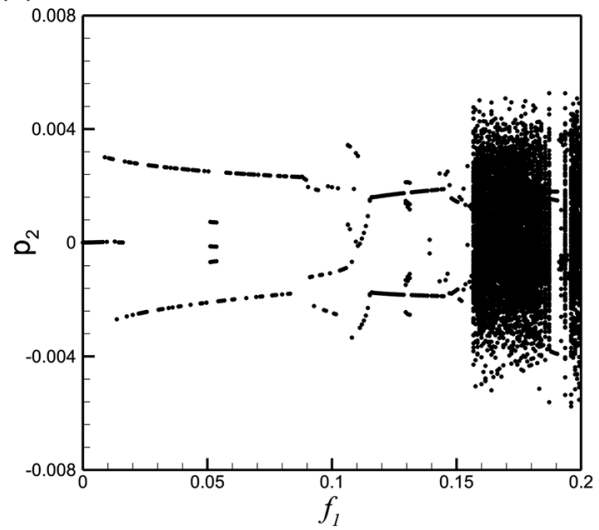

(f)

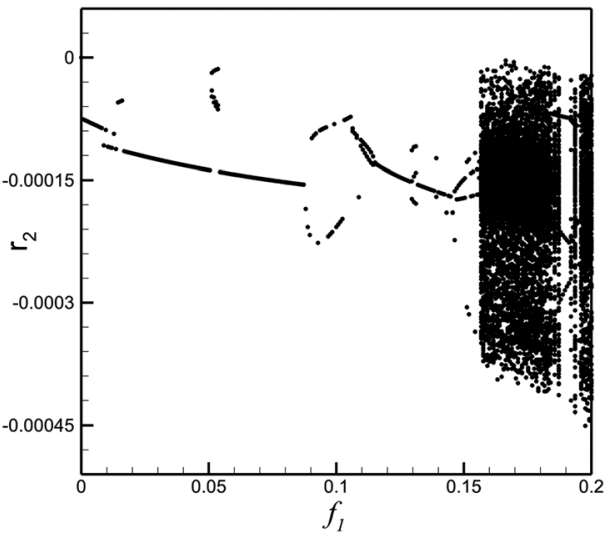

Fig. 2 Bifurcation diagrams of the Poincare sections for increasing forcing amplitude on the system with $c=1.35$ : (a) and $(b)$ the first two generalized coordinates of the transverse motion; $(c)$ and $(d)$ the first two generalized coordinates of the lateral motion; and $(e)$ and $(f)$ the first two generalized coordinates of the longitudinal motion 

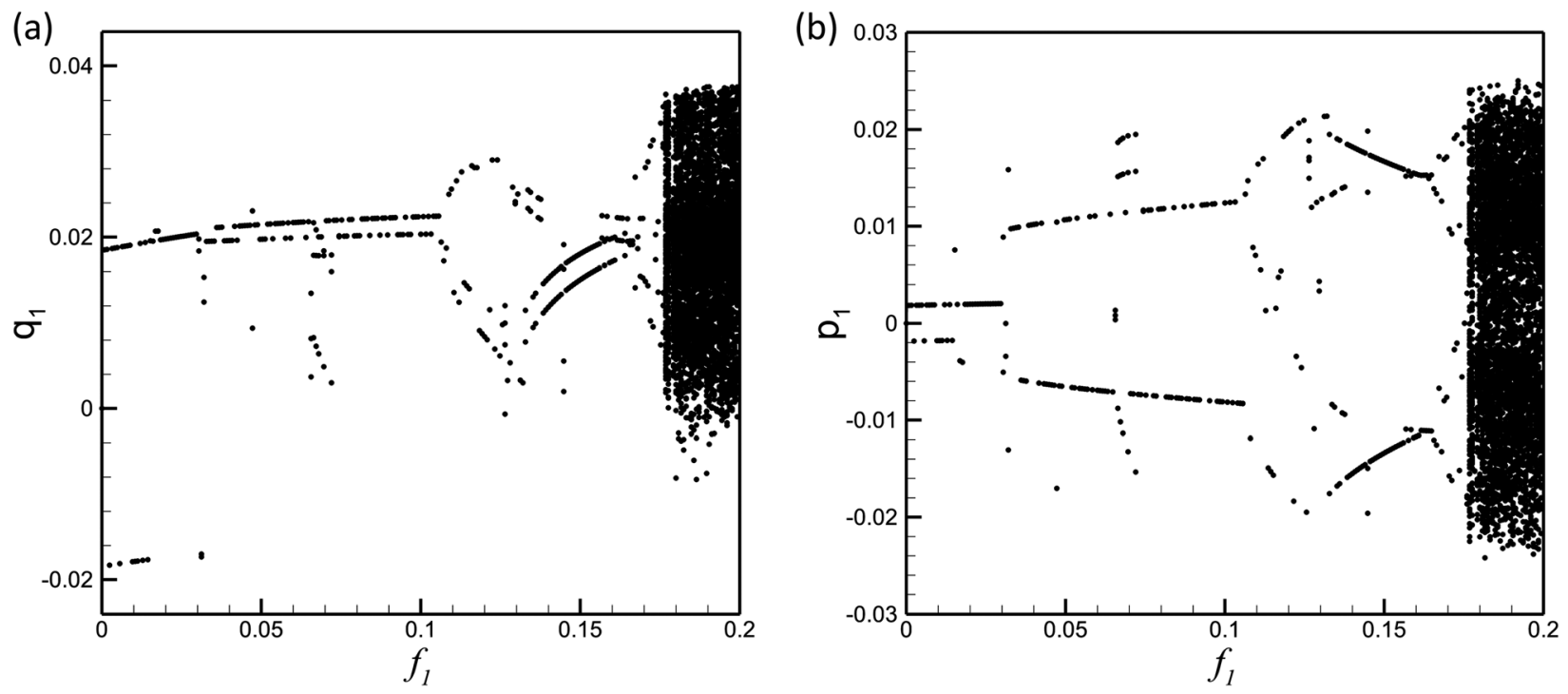

Fig. 3 Bifurcation diagrams of the Poincaré sections for increasing forcing amplitude on the system with $c=1.50$ : (a) and (b) the first generalized coordinate of the transverse and lateral motions, respectively

[38]. Sectioning these time traces at every period of the external excitation frequency results in the bifurcation diagrams of Poincaré sections.

\section{Numerical Results for Nonlinear Global Dynamics}

The numerical results presented in this section are obtained by conducting direct time-integration upon the discretized equations of motion. This procedure results in the time traces of the generalized coordinates of the system for certain system parameters. It should be noted that the integration in time is continued until $t=3000$ dimensionless seconds and the first $70 \%$ of the response has been excluded in order to remove any possible transient effects. Poincaré sections are attained by sectioning the phasespace in every period of the excitation frequency; the bifurcation diagrams of Poincaré sections are constructed by varying either the excitation amplitude or the axial speed as the bifurcation parameter. In each case, the final state of the system is taken as the initial condition for the new bifurcation parameter so as to

(a)

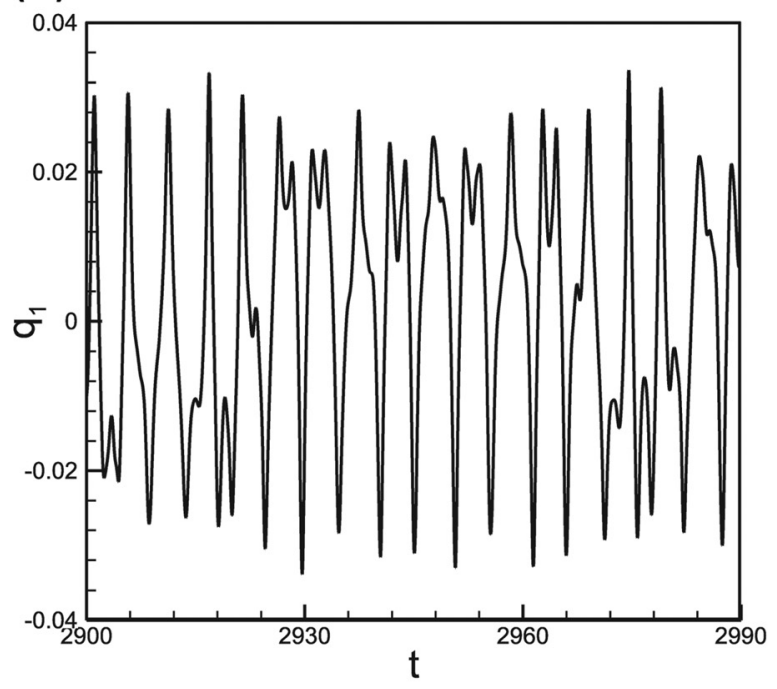

keep track of a certain attractor. The excitation frequency is set to 1.2 and $\alpha$ is chosen equal to one for all the cases studied in this section. It is worthwhile noting that for a system with $\alpha=1$ (i.e., $I_{y}=I_{z}$ ), the system (in the absence of external forces) becomes axisymmetric with respect to the centerline of the beam. This axisymmetric configuration yields the same linear natural frequencies for the transverse and lateral motions - the system possesses oneto-one internal resonances. In what follows, it is implied that response and amplitude are with respect to the $q_{1}$ motion and the amplitude of the $q_{1}$ motion where it is sectioned, respectively.

3.1 Forcing Amplitude as the Bifurcation Parameter. The forcing amplitude, $f_{1}$, is chosen as the bifurcation parameter in this section in order to construct the bifurcation diagrams; the system is also excited in the lateral (i.e., $z$ ) direction by choosing $f_{2}=0.1 f_{1}$. The bifurcation diagrams are constructed for two axial speeds, namely, $c=1.35$ and 1.50 , and plotted in Figs. 2 and 3, respectively-these axial speeds are in the supercritical regime.

(b)

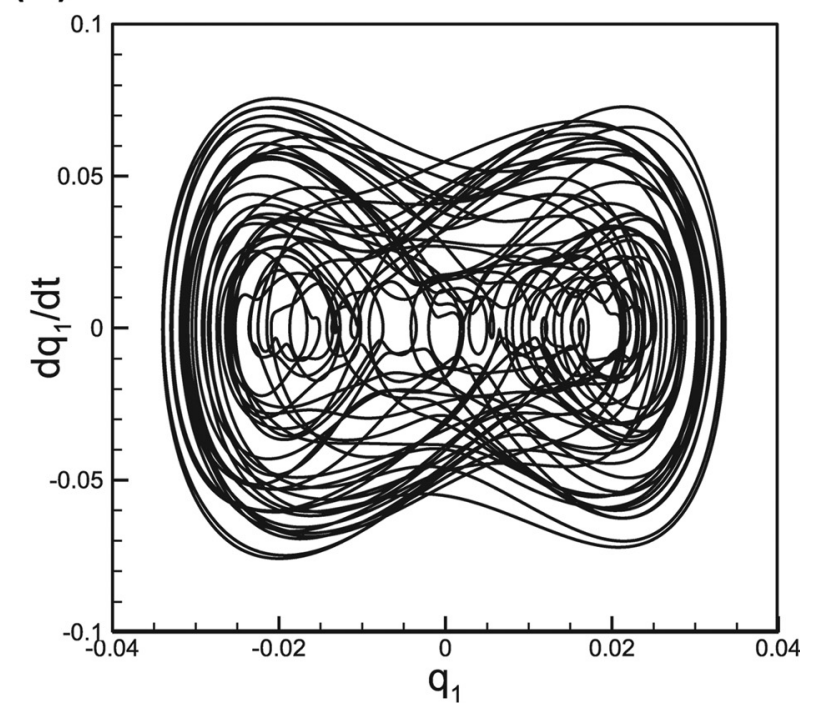

Fig. 4 Chaotic motion of the system of Fig. 2 at $f_{1}=0.1680$ : (a) and (b) time trace and phase-plane diagram of the $q_{1}$ motion, respectively 

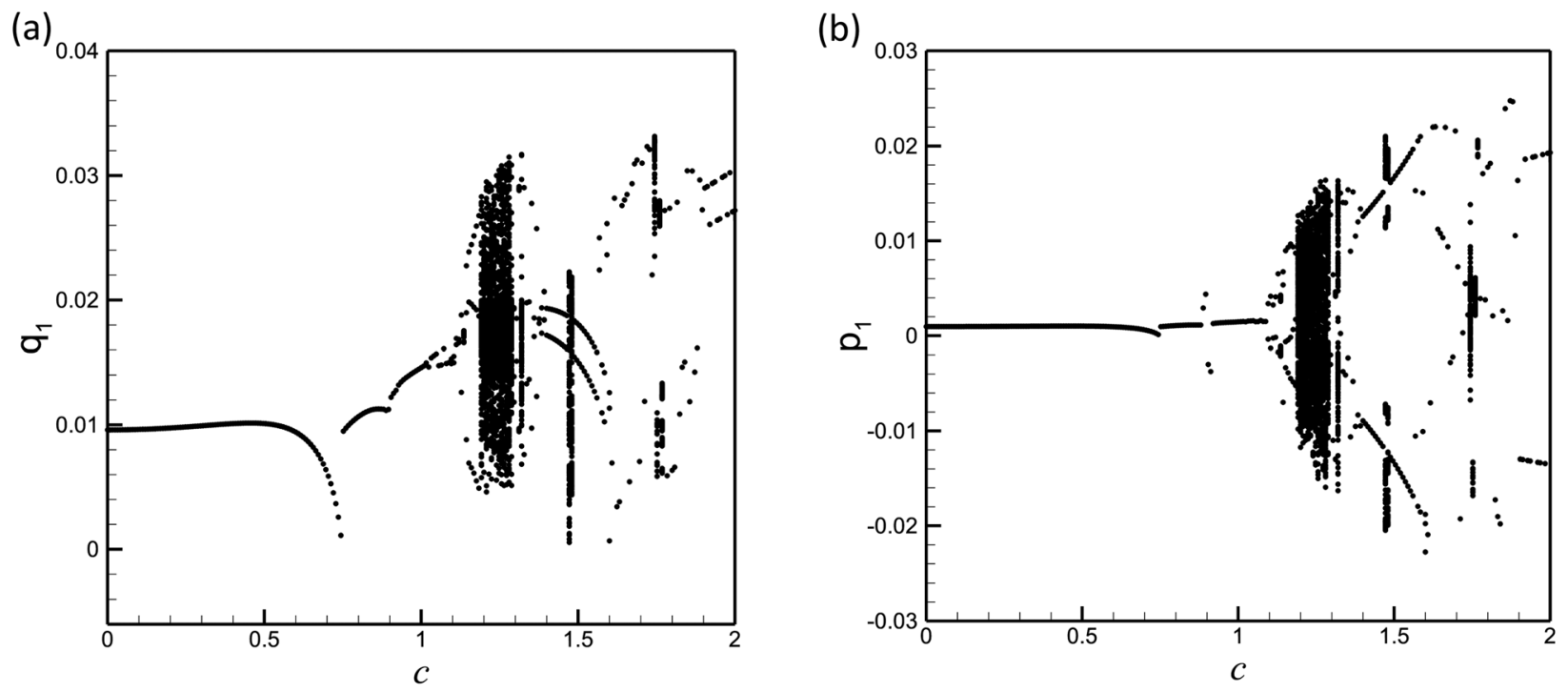

Fig. 5 Bifurcation diagrams of the Poincare sections for increasing axial speed of the system with $f_{1}=0.15$ : $(a)$ and $(b)$ the first generalized coordinate of the transverse and lateral motions, respectively

The other dimensionless system parameters are selected as the following: $v_{1}=33.526, v_{f}=0.1732, \zeta=20, \gamma=0.00000749, \alpha=1$, $\Omega=1.2$, and $\mu=0.04$.

Figure 2 shows the bifurcation diagrams of the system for the case with $c=1.35$, for the first two generalized coordinates of the transverse $\left(q_{1}\right.$ and $\left.q_{2}\right)$, lateral $\left(p_{1}\right.$ and $\left.p_{2}\right)$, and longitudinal $\left(r_{1}\right.$ and $r_{2}$ ) motions. As seen in Fig. 2(a), the response amplitude is nonzero for $f_{1}=0$, showing that the system is diverged to a nontrivial equilibrium configuration, since the axial speed belongs to the supercritical regime. The figure shows that the amplitude of the pure periodic motion increases with increasing forcing amplitude in the range of [0 0.0080]. Two limit cycle attractors coexist in the forcing amplitude range of [0.0088 0.0872$]$, where the system jumps repeatedly from one to another. This structure can be seen in other bifurcation diagrams, namely, Figs. $2(b)-2(d)$ as well. The same scenario happens for the forcing amplitude range of $\left[\begin{array}{ll}0.0880 & 0.1056\end{array}\right]$, with different motion amplitudes for the two pure periodic attractors. A period-2 attractor prevails thereafter for a noticeably large interval, i.e., [0.1064 0.1552], with a short- lived window of period-6 motion in between. By further incrementing the forcing amplitude, a direct transition from a period-2 to chaotic motion can be observed at $f_{1}=0.1568$. The motion is dominantly chaotic for higher values of the forcing amplitude until $f_{1}=0.2000$ is reached. Typical characteristics of a chaotic motion at $f_{1}=0.1680$ are shown in Fig. 4 .

The bifurcation diagrams of the system with $c=1.50$ are illustrated in Fig. 3 (the results are shown only for the first generalized coordinate of the transverse and lateral motions). For this axial speed, a dynamical behavior similar to that of the system of Fig. 2 emerges, where the most features, such as the coexisting attractors and bifurcations, are preserved. However, due to increased axial speed, the number of higher-order bifurcations increases, the overall amplitude of the motion increases, the chaotic motion occurs at higher forcing amplitudes, and the chaotic region becomes narrower. Comparing Figs. 2 and 3, it can be concluded that higher axial speed does not necessarily guarantee more complex dynamics with more chaotic attractors.

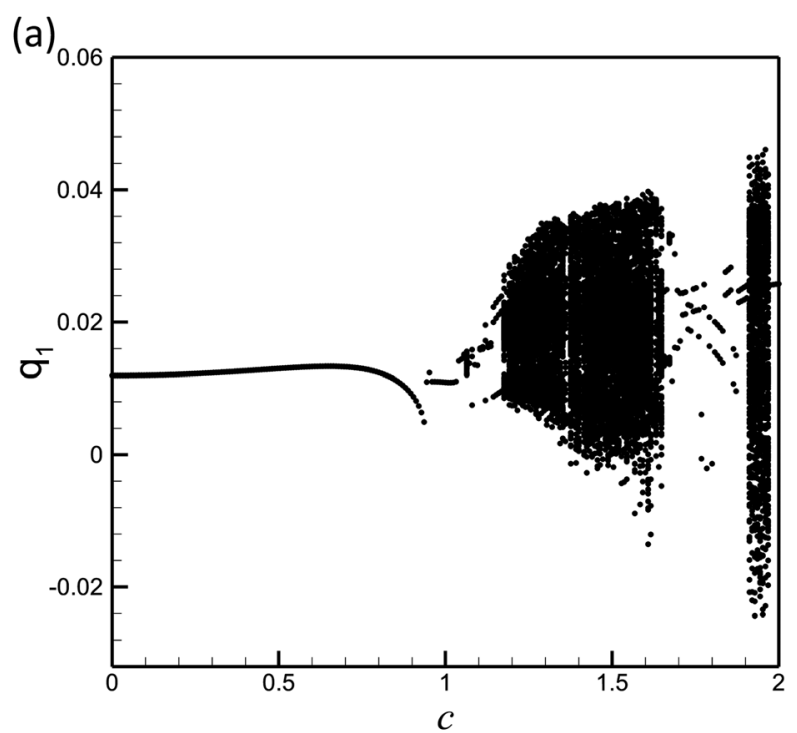

(b)

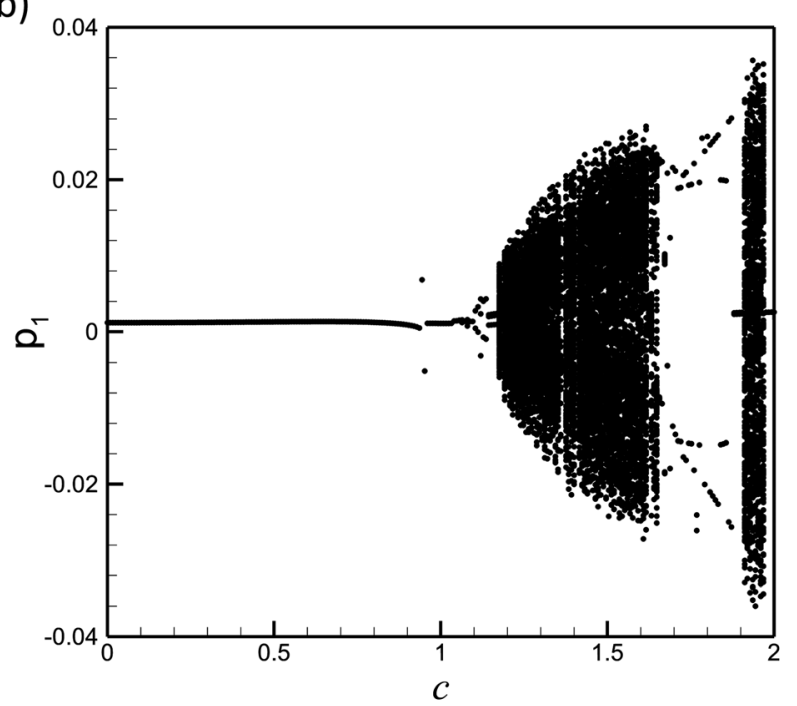

Fig. 6 Bifurcation diagrams of the Poincaré sections for increasing axial speed of the system with $f_{1}=0.20$ : $(a)$ and $(b)$ the first generalized coordinate of the transverse and lateral motions, respectively 
3.2 Axial Speed as the Bifurcation Parameter. The bifurcation diagrams of this section are constructed by varying the axial speed as the bifurcation parameter. In particular, two forcing amplitudes are selected, namely, $f_{1}=0.15$ and 0.20 , and the corresponding bifurcation diagrams are plotted in Figs. 5 and 6, respectively; $f_{2}$ is chosen equal to $0.1 f_{1}$. The following dimensionless system parameters are chosen for the numerical analysis of this section: $v_{1}=33.526, v_{f}=0.1732, \zeta=20, \gamma=0.00000749, \alpha=1$, $\Omega=1.2$, and $\mu=0.04$.

The bifurcation diagrams for the system with $f_{1}=0.15$ are plotted in Fig. 5. Figure 5(a) shows that, as the axial speed is increased from zero, there is a small growth in the motion amplitude which is continued by a sharp decay until $c=0.752$ is reached, corresponding to the first jump in the system response. After a range of pure periodic motions, the first period-2 attractor occurs at $c=1.096$. This period-2 motion is very short-lived and by a small increment in $c$ the system experiences a short burst of period-3 motions in the interval [1.144 1.184]. After this intermittent period-3 dynamics, the motion becomes chaotic at $c=1.192$ which lasts until $c=1.296$ is hit, where the motion becomes period- 2 once again. This robust period- 2 motion prevails until $c=1.608$ is reached, where it is replaced by two coexisting periodic attractors. The response of the system is characterized by this

(a)

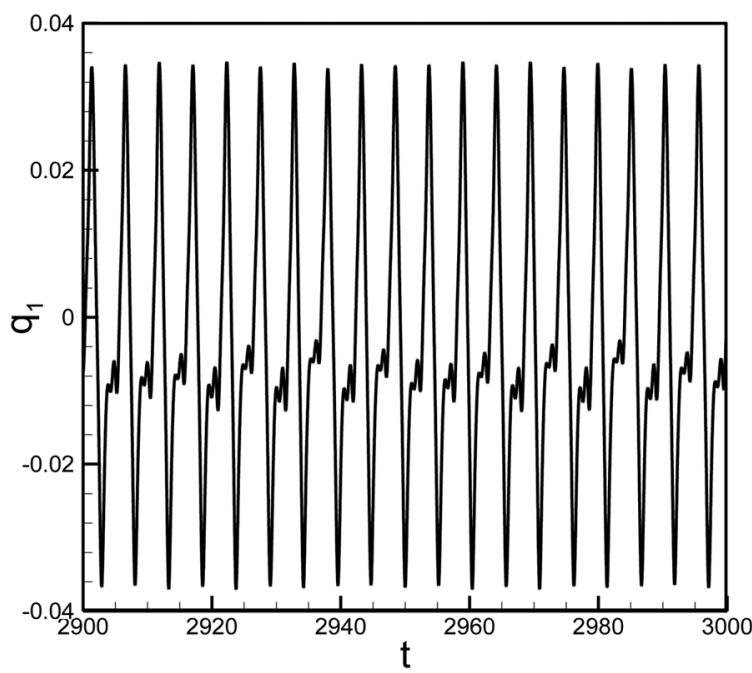

(c)

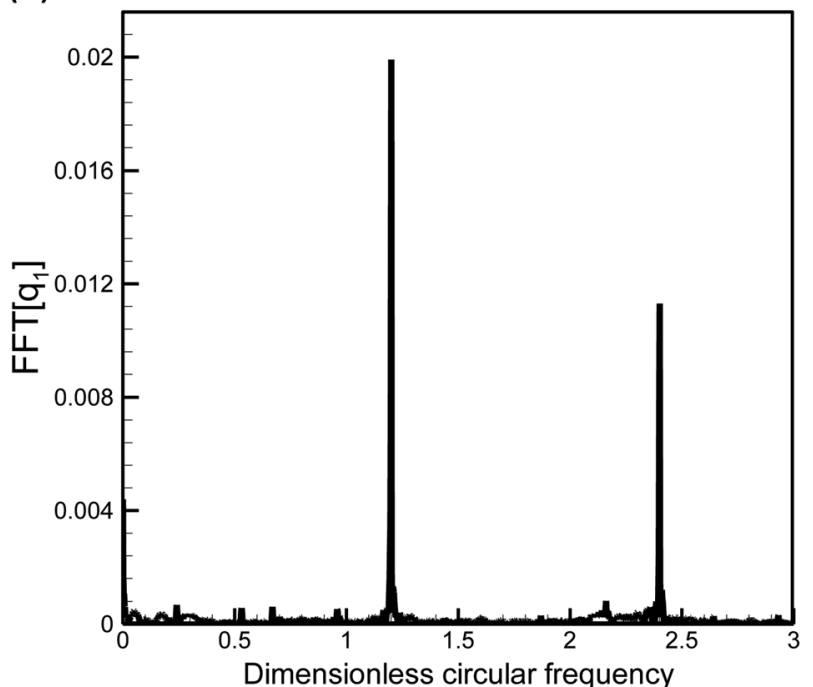

two coexisting pure periodic attractors thereafter until $c=2.000$, with intermittent chaotic and quasi-periodic attractors in the interval [1.744 1.768]. The characteristics of a quasi-periodic attractor at $c=1.760$ are shown in Fig. 7 .

The bifurcation diagrams regarding a higher value of the forcing amplitude (i.e., $f_{1}=0.20$ ), compared to the system of Fig. 5, are depicted in Fig. 6. As shown in Fig. 6(a), the system displays a pure periodic motion with almost a constant amplitude in the axial speed range of $[0.0000 .800]$. Increasing the axial speed further, the amplitude of the motion decreases until $c=0.944$, where the first jump occurs. Increasing the axial speed even further, the system displays short bursts of quasi-periodic, period-2, and period-3 motions in the interval [1.056 1.168]. Chaotic motion prevails for a noticeably large interval, i.e., [1.176 1.648], continued by a period- 2 attractor. After the intermittent period- 2 dynamics, the motion becomes chaotic once again which lasts for a short interval. Finally, the system regains the original period at $c=1.976$ and maintains that period until $c=2.000$ is reached.

Comparing Figs. 5 and 6, one may draw the general conclusion that, due to increased forcing amplitude, the number of higherorder bifurcations decreases in the cascade of bifurcations and the chaotic regions become wider. Moreover, as a general trend, it can be inferred that the first saddle-node bifurcation (which

(b)

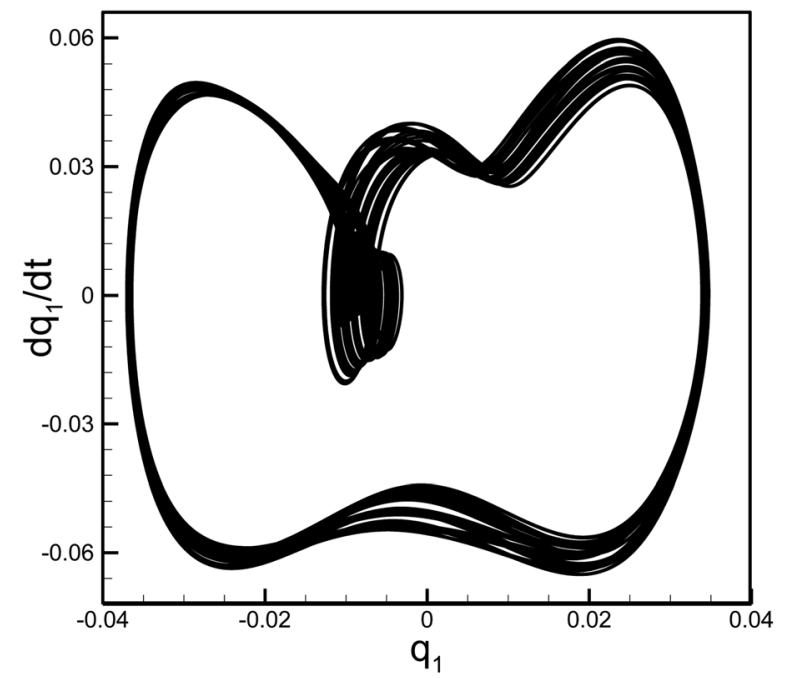

(d)

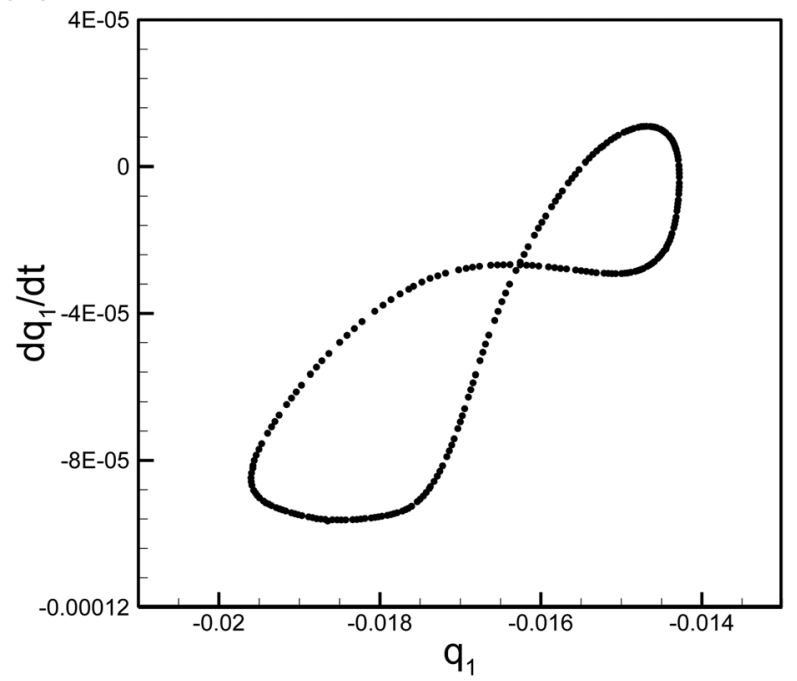

Fig. 7 Quasi-periodic motion of the system of Fig. 5 at $c=1.760$ : (a)-(d) time trace, phase-plane diagram, FFT, and Poincaré map of the $q_{1}$ motion, respectively 
appears as a jump in the bifurcation diagram) is delayed due to increased forcing amplitude.

\section{Effect of Viscoelasticity on the Global Dynamics of the System}

In this section, the bifurcation diagrams obtained employing a viscoelastic model are compared to those obtained using an elastic model (by setting $\zeta=\gamma=0$ in Eqs. (8)-(10)), so as to highlight the effect of internal energy dissipation mechanism (the Kelvin-Voigt model) on the nonlinear global dynamics of the system. In particular, the bifurcation diagrams corresponding to the first generalized coordinate of the transverse and lateral motions are constructed for both the viscoelastic and elastic systems, as either the forcing amplitude or the axial speed is varied as the bifurcation parameter. The following dimensionless system parameters are chosen for the numerical analysis: $v_{1}=33.526, v_{f}=0.1732, \alpha$ $=1, \Omega=1.2$, and $\mu=0.04$. Moreover, $\zeta=20$ and $\gamma$ $=0.00000749$ are used for the viscoelastic model (i.e., in the presence of the Kelvin-Voigt energy dissipation mechanism), while $\zeta$ $=\gamma=0$ is used for the elastic model (i.e., in the absence of the Kelvin-Voigt energy dissipation mechanism).

Figure 8 shows the bifurcation diagrams of both the viscoelastic and elastic systems when the forcing amplitude is varied as the bifurcation parameter and the axial speed is set to $c=1.50$. The figure reveals that the viscoelastic system displays much simpler dynamics. It is observed that due to the presence of an internal energy dissipation mechanism, some of the early chaotic regions for the elastic systems are changed to periodic regions for the viscoelastic system. Moreover, in general, for the viscoelastic system, the chaotic attractors occur at higher forcing amplitudes and chaotic regions disappear or become narrower.

Figure 9 shows the comparison between the bifurcation diagrams of the viscoelastic and elastic systems when the axial speed is varied as the bifurcation parameter and the forcing amplitude is set to $f_{1}=0.15$. It is seen that most of the chaotic regions are vanished due to the presence of the internal energy dissipation mechanism. It is also observed that the first saddle-node bifurcation occurs at lower axial speeds for the viscoelastic system. Hence, one may draw the general conclusion that the viscoelastic system (a)

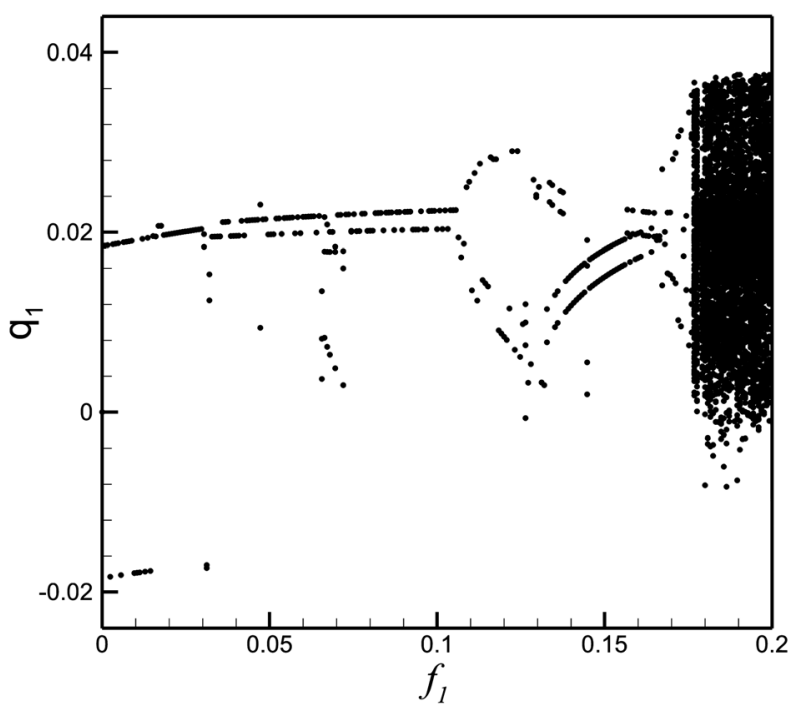

(c)

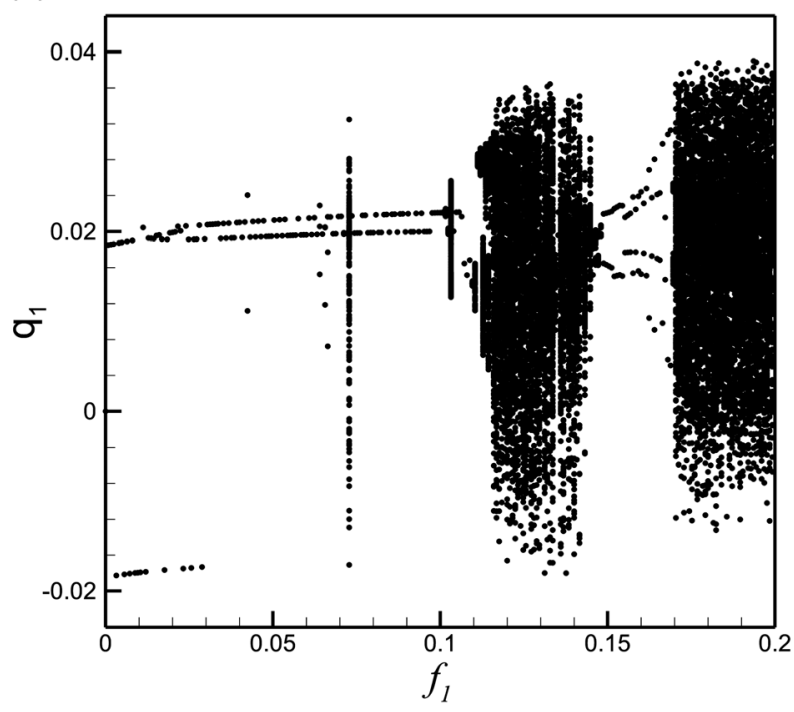

(b)

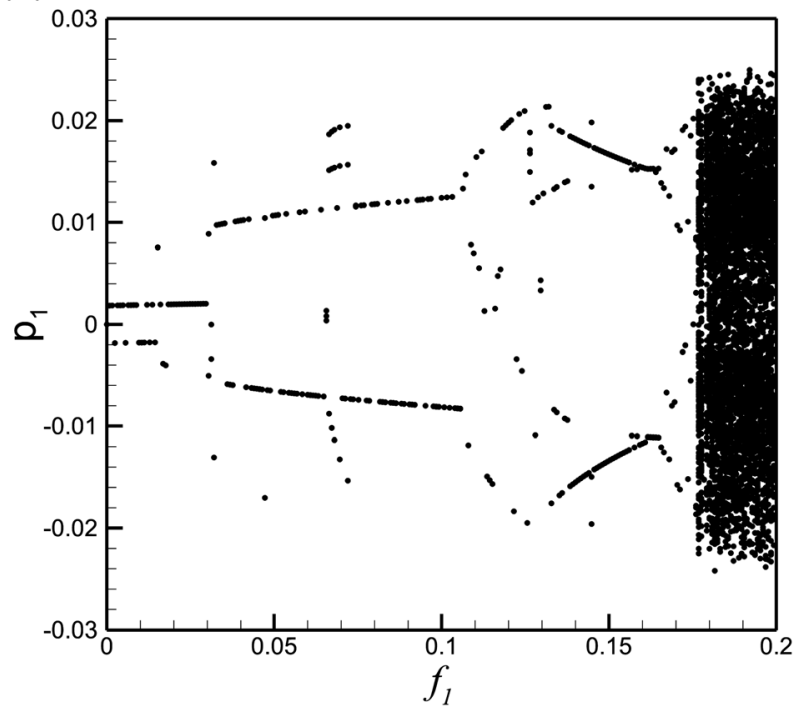

(d)

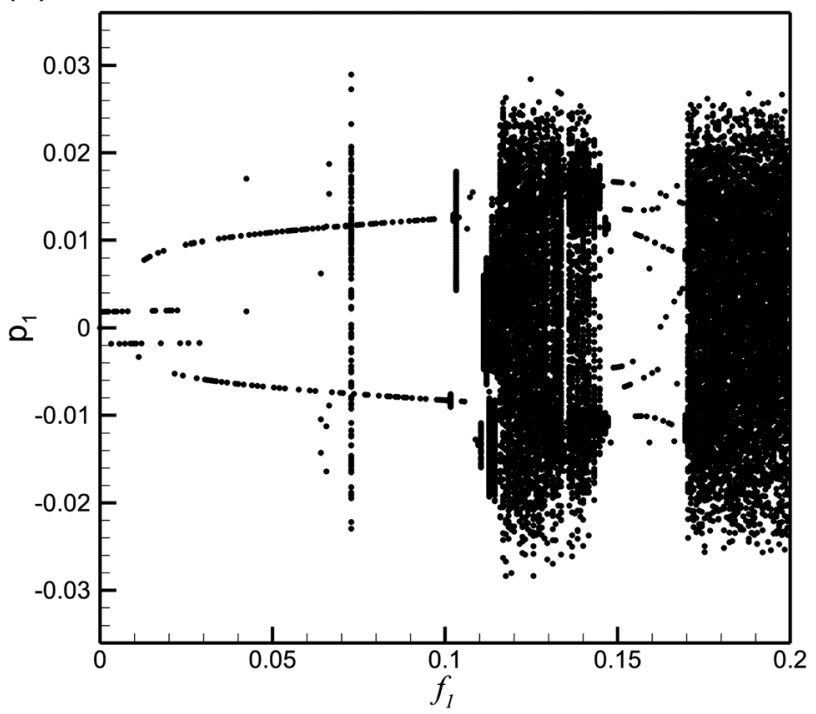

Fig. 8 Bifurcation diagrams of the Poincaré sections for increasing forcing amplitude on the system with $c=1.50$ : (a) and (b) correspond to the first generalized coordinate of the transverse and lateral motions of the viscoelastic system and (c) and $(d)$ are the counterparts of $(a)$ and $(b)$ for the elastic system; $f_{2}=0.1 f_{1}$ 
(a)

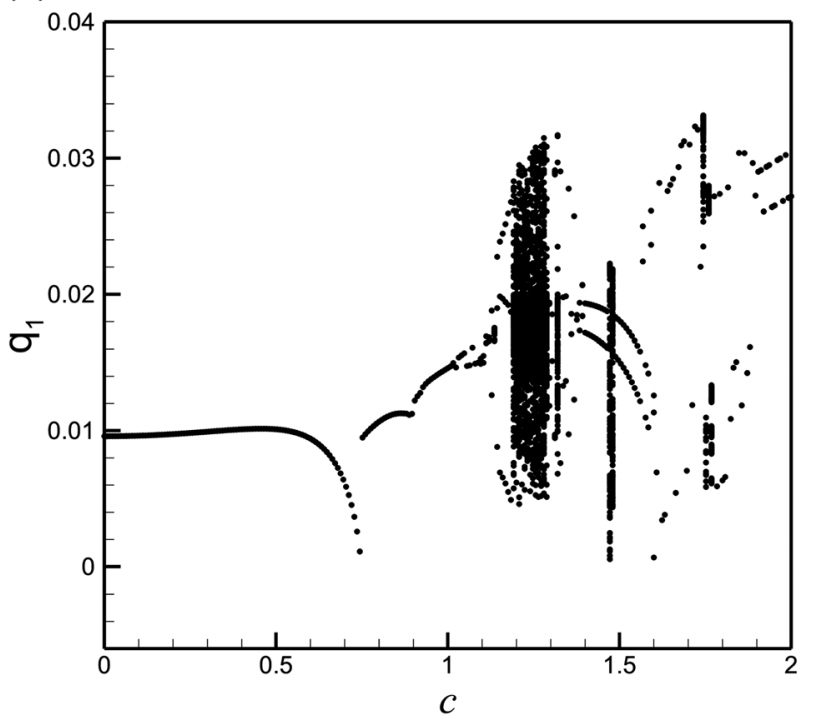

(c)

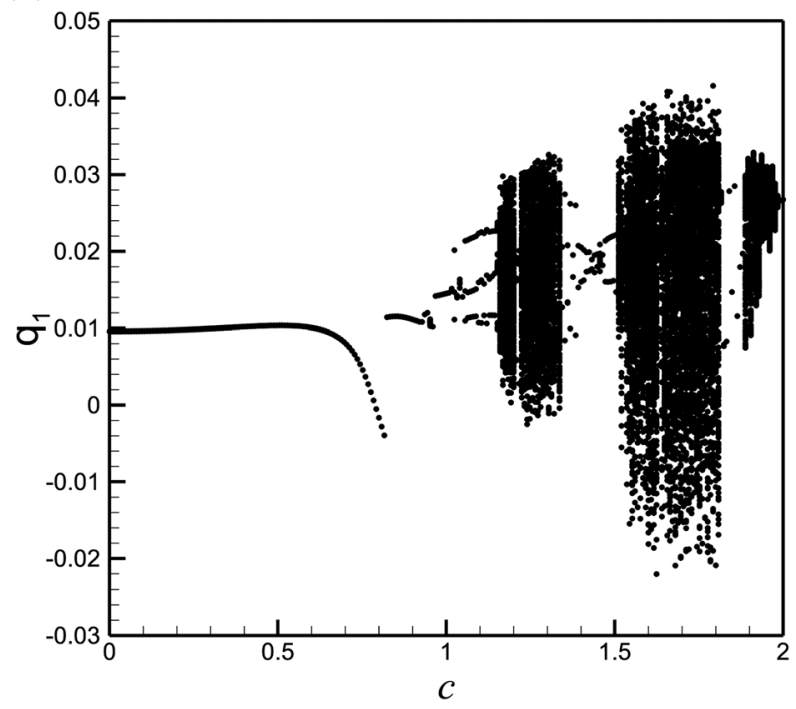

(b)

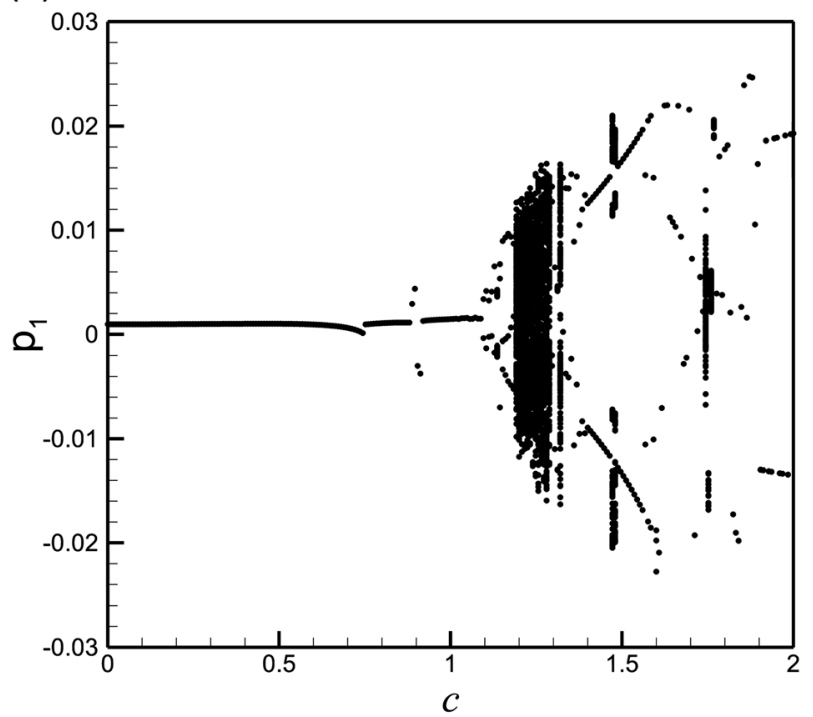

(d)

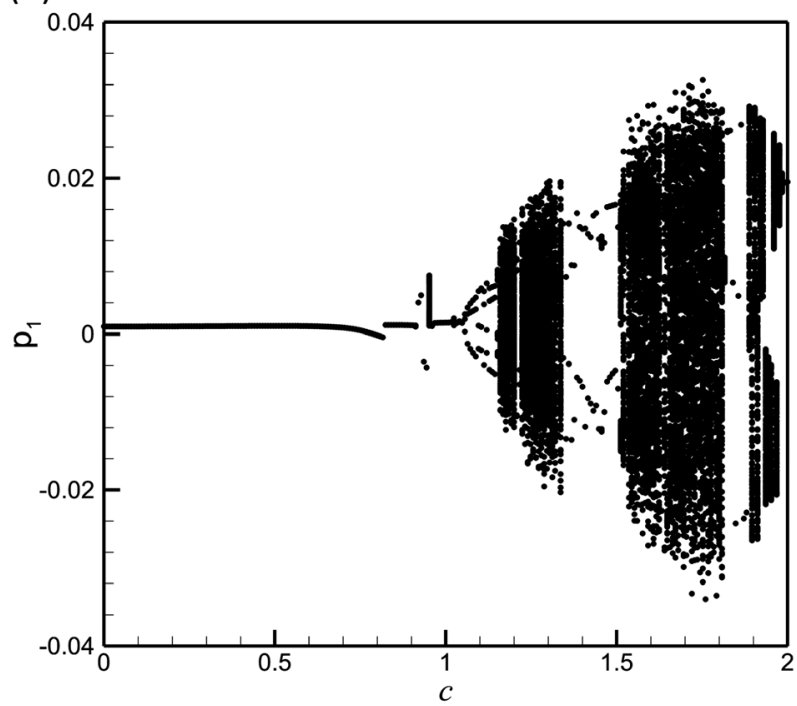

Fig. 9 Bifurcation diagrams of the Poincaré sections for increasing axial speed for the system with $f_{1}=0.15$ : $(a)$ and $(b)$ correspond to the first generalized coordinate of the transverse and lateral motions of the viscoelastic system and (c) and (d) are the counterparts of $(a)$ and $(b)$ for the elastic system; $f_{2}=0.1 f_{1}$

exhibits much simpler dynamics with less complex and coexisting attractors and narrower chaotic regions.

\section{Conclusions}

The nonlinear global dynamics of a three-dimensional axially moving viscoelastic beam has been investigated numerically. The nonlinear partial differential equations governing the motion of the system in the longitudinal, transverse, and lateral directions were derived by means of Hamilton's principle and Kelvin-Voigt viscoelastic model. The Galerkin scheme was used to discretize the partial differential equations of motion into a set of secondorder nonlinear ordinary differential equations. The modified Rosenbrock method was employed in order to conduct direct time-integration upon the discretized equations of motion, resulting in the time-varying generalized coordinates. The bifurcation diagrams of Poincare sections were then constructed by sectioning the time trances of the generalized coordinates in every period of the excitation frequency.

Examining the nonlinear dynamics of the system, for the case when the forcing amplitude is chosen as the bifurcation parameter, revealed that a higher axial speed does not necessarily result in more chaotic regions. However, the results for the case when the axial speed is the bifurcation parameter showed that the chaotic regions increase with increasing forcing amplitude. Moreover, the chaotic regions become wider and the chaotic motion occurs at lower axial speeds.

Comparing the results obtained for both the viscoelastic and elastic systems revealed that, in general, the system displays simpler dynamics due to the presence of the internal energy dissipation mechanism. It was shown that the number of chaotic regions as well as higher-order bifurcations decreases for the viscoelastic model and the chaotic regions become narrower.

\section{Acknowledgment}

The financial support to this research by the start-up grant of the University of Wollongong is gratefully acknowledged.

\section{References}

[1] Yang, T.-Z., Yang, X., Chen, F., and Fang, B., 2013, "Nonlinear Parametric Resonance of a Fractional Damped Axially Moving String," ASME J. Vib. Acoust., 135(6), p. 064507. 
[2] Sandilo, S. H., and van Horssen, W. T., 2012, "On Boundary Damping for an Axially Moving Tensioned Beam," ASME J. Vib. Acoust., 134(1), p. 011005.

[3] Ding, H., and Zu, J. W., 2014, "Steady-State Responses of Pulley-Belt Systems With a One-Way Clutch and Belt Bending Stiffness," ASME J. Vib. Acoust., 136(4), p. 041006

[4] Ding, H., Yan, Q.-Y., and Zu, J. W., 2014, "Chaotic Dynamics of an Axially Accelerating Viscoelastic Beam in the Supercritical Regime," Int. J. Bifurcation Chaos, 24(5), p. 1450062.

[5] Mockensturm, E. M., and Guo, J., 2005, "Nonlinear Vibration of Parametrically Excited, Viscoelastic, Axially Moving Strings," ASME J. Appl. Mech., 72(3), pp. 374-380.

[6] Ghayesh, M. H., 2010, "Parametric Vibrations and Stability of an Axially Accelerating String Guided by a Non-Linear Elastic Foundation," Int. J. NonLinear Mech., 45(4), pp. 382-394.

[7] Ghayesh, M. H., 2011, "On the Natural Frequencies, Complex Mode Functions, and Critical Speeds of Axially Traveling Laminated Beams: Parametric Study," Acta Mech. Solida Sin., 24(4), pp. 373-382.

[8] Ghayesh, M. H., 2012, "Coupled Longitudinal-Transverse Dynamics of an Axially Accelerating Beam," J. Sound Vib., 331(23), pp. 5107-5124.

[9] Mote, C. D., Jr., 1965, "A Study of Band Saw Vibrations," J. Franklin Inst., 279(6), pp. 430-444

[10] Wickert, J. A., 1992, “Non-Linear Vibration of a Traveling Tensioned Beam," Int. J. Non-Linear Mech., 27(3), pp. 503-517.

[11] Pakdemirli, M., and Ulsoy, A. G., 1997, "Stability Analysis of an Axially Accelerating String," J. Sound Vib., 203(5), pp. 815-832.

[12] Özkaya, E., and Pakdemirli, M., 2000, "Vibrations of an Axially Accelerating Beam With Small Flexural Stiffness," J. Sound Vib., 234(3), pp. 521-535.

[13] Öz, H. R., Pakdemirli, M., and Boyacı, H., 2001, "Non-Linear Vibrations and Stability of an Axially Moving Beam With Time-Dependent Velocity," Int. J. Non-Linear Mech., 36(1), pp. 107-115.

[14] Marynowski, K., and Kapitaniak, T., 2007, "Zener Internal Damping in Modelling of Axially Moving Viscoelastic Beam With Time-Dependent Tension,' Int. J. Non-Linear Mech., 42(1), pp. 118-131.

[15] Stylianou, M., and Tabarrok, B., 1994, "Finite Element Analysis of an Axially Moving Beam, Part II: Stability Analysis," J. Sound Vib., 178(4), pp. 455-481.

[16] Chakraborty, G., and Mallik, A. K., 1999, "Stability of an Accelerating Beam," J. Sound Vib., 227(2), pp. 309-320.

[17] Zhang, N.-H., and Chen, L.-Q., 2005, "Nonlinear Dynamical Analysis of Axially Moving Viscoelastic Strings," Chaos, Solitons Fractals, 24(4), pp. 1065-1074.

[18] Chen, L.-Q., and Chen, H., 2010, "Asymptotic Analysis on Nonlinear Vibration of Axially Accelerating Viscoelastic Strings With the Standard Linear Solid Model,” J. Eng. Math., 67(3), pp. 205-218.

[19] Tang, Y.-Q., Chen, L.-Q., and Yang, X.-D., 2009, "Nonlinear Vibrations of Axially Moving Timoshenko Beams Under Weak and Strong External Excitations," J. Sound Vib., 320(4-5), pp. 1078-1099.

[20] Ding, H., and Chen, L.-Q., 2010, "Galerkin Methods for Natural Frequencies of High-Speed Axially Moving Beams," J. Sound Vib., 329(17), pp. 3484-3494

[21] Chen, L.-Q., and Ding, H., 2010, "Steady-State Transverse Response in Coupled Planar Vibration of Axially Moving Viscoelastic Beams," ASME J. Vib. Acoust., 132(1), p. 011009.
[22] Ding, H., and Chen, L.-Q., 2008, "Stability of Axially Accelerating Viscoelastic Beams: Multi-Scale Analysis With Numerical Confirmations," Eur. J. Mech.-A/ Solids, 27(6), pp. 1108-1120.

[23] Ravindra, B., and Zhu, W. D., 1998, "Low-Dimensional Chaotic Response of Axially Accelerating Continuum in the Supercritical Regime," Arch. Appl. Mech., 68(3-4), pp. 195-205.

[24] Huang, J.-S., Fung, R.-F., and Lin, C.-H., 1995, "Dynamic Stability of a Moving String Undergoing Three-Dimensional Vibration," Int. J. Mech. Sci., 37(2), pp. $145-160$.

[25] Chen, L. H., Zhang, W., and Yang, F. H., 2010, "Nonlinear Dynamics of Higher-Dimensional System for an Axially Accelerating Viscoelastic Beam With In-Plane and Out-of-Plane Vibrations," J. Sound Vib., 329(25), pp. $5321-5345$.

[26] Ghayesh, M., 2012, "Subharmonic Dynamics of an Axially Accelerating Beam,” Arch. Appl. Mech., 82(9), pp. 1169-1181.

[27] Ghayesh, M. H., Kazemirad, S., and Reid, T., 2012, "Nonlinear Vibrations and Stability of Parametrically Exited Systems With Cubic Nonlinearities and Internal Boundary Conditions: A General Solution Procedure," Appl. Math. Modell., 36(7), pp. 3299-3311.

[28] Ghayesh, M. H., Kazemirad, S., and Darabi, M. A., 2011 "A General Solution Procedure for Vibrations of Systems With Cubic Nonlinearities and Nonlinear/ Time-Dependent Internal Boundary Conditions," J. Sound Vib., 330(22), pp. $5382-5400$.

[29] Ghayesh, M., and Farokhi, H., 2014, "Post-Buckling Dynamics of Timoshenko Microbeams Under Axial Loads,” Int. J. Dyn. Control.

[30] Ghayesh, M. H., Kafiabad, H. A., and Reid, T., 2012, "Sub- and Super-Critical Nonlinear Dynamics of a Harmonically Excited Axially Moving Beam," Int. J. Solids Struct., 49(1), pp. 227-243.

[31] Ghayesh, M. H., and Farokhi, H., 2015, "Coupled Longitudinal-TransverseRotational Behaviour of Shear Deformable Microbeams," Composites Part B, 77, pp. 319-328.

[32] Gholipour, A., Farokhi, H., and Ghayesh, M., 2014, "In-Plane and Out-of-Plane Nonlinear Size-Dependent Dynamics of Microplates," Nonlinear Dyn., 79(3), pp. 1771-1785.

[33] Ghayesh, M. H., and Farokhi, H., 2015, “Thermo-Mechanical Dynamics of Three-Dimensional Axially Moving Beams," Nonlinear Dyn. 80(3), pp. $1643-1660$.

[34] Ghayesh, M. H., Farokhi, H., and Alici, G., 2015, "Internal Energy Transfer in Dynamical Behaviour of Slightly Curved Shear Deformable Microplates," ASME J. Comput. Nonlinear Dyn. (in press).

[35] Ghayesh, M., 2012, "Stability and Bifurcations of an Axially Moving Beam With an Intermediate Spring Support," Nonlinear Dyn., 69(1-2), pp. 193-210.

[36] Ghayesh, M. H., 2011, "Nonlinear Forced Dynamics of an Axially Moving Viscoelastic Beam With an Internal Resonance," Int. J. Mech. Sci., 53(11), pp. 1022-1037.

[37] Ghayesh, M. H., 2012, "Nonlinear Dynamic Response of a Simply-Supported Kelvin-Voigt Viscoelastic Beam, Additionally Supported by a Nonlinear Spring," Nonlinear Anal.: Real World Appl., 13(3), pp. 1319-1333.

[38] Ghayesh, M. H., and Farokhi, H., 2015, "Chaotic Motion of a Parametrically Excited Microbeam,” Int. J. Eng. Sci., 96, pp. 34-45. 